\title{
Myrica faya: A New Source of Antioxidant Phytochemicals
}

\author{
Vítor Spínola, ${ }^{\dagger}$ Eulogio J. Llorent-Martínez, ${ }^{\dagger \neq}{ }^{\dagger}$ Sandra Gouveia, ${ }^{\S}$ and Paula C. Castilho*, ${ }^{\dagger}$ \\ ${ }^{\dagger}$ Centro de Química da Madeira (CQM), Campus Universitário da Penteada, 9000-390 Funchal, Portugal \\ ${ }^{*}$ Department of Physical and Analytical Chemistry, Faculty of Experimental Sciences, University of Jaén, Campus Las Lagunillas S/N, \\ E-23071 Jaén, Spain \\ ${ }^{\S}$ Department of Chemistry, Umeå University, 90187 Umeå, Sweden
}

ABSTRACT: Myrica faya is a fruit tree endemic of the Macaronesia (Azores, Madeira, and Canary Island), and its edible fruits are known as "amorinhos" (little loves), bright red to purple berries, used fresh and in jams and liquors. The phenolic composition and antioxidant capacity of leaves and berries from $M$. faya are presented here for the first time. The screening of phytochemical compounds was carried out using high-performance liquid chromatography with online UV and electrospray ionization mass spectrometric detection (HPLC-DAD-ESI-MS ${ }^{n}$ ). There were 55 compounds characterized, mostly galloyl esters of flavonoids and phenolic acids; 26 of the identified compounds (anthocyanins, isoflavonoids, lignans, terpenes, fatty acids, and phenylethanoids) have not been reported in Myrica genus so far. From the data presented here, it can be concluded that faya berries represent a rich source of cyanidin-3-glucoside, flavonoids, and vitamin C. In fact, higher antioxidant activity than that of the well-known Myrica rubra berries (Chinese bayberry) has been observed.

KEYWORDS: Myrica faya, phenolic compounds, HPLC-DAD-ESI/MS ${ }^{n}$, vitamin C, antioxidant activity

\section{INTRODUCTION}

Under oxidative stress, the human body produces more reactive oxygen species than enzymatic and nonenzymatic antioxidants. This imbalance leads to cell damage and facilitates the development of degenerative diseases, including cardiovascular diseases, cancers, and Alzheimer's disease. ${ }^{1}$ Fruits and vegetables provide a variety of phytochemicals, including phenolic compounds, a class of secondary metabolites, synthesized by the plants during normal development, and in response to stress conditions. Polyphenols (such as phenolic acids and flavonoids) present high antioxidant activity and, therefore, many health promoting effects (anti-inflammatory, antiallergic, antiaging, and anticarcinogenic activities), serving as a type of preventive medicine. ${ }^{2,3}$ Hence, research on the chemical composition of already-known medicinal plants and on new plants with potential antioxidant value is currently being performed throughout the world.

Laurisilva, the Madeira (Portugal) laurel forest, is a subtropical forest with a very rich flora and is considered the most important remnants of the evergreen laurel forest from the Tertiary period. It was declared a biogenetic reserve of the European Council and world natural patrimony under the protection of UNESCO in 1999. The plants present in this forest are endemic to Macaronesia, and are protected species. They are well-studied and characterized from the botanical point of view, but their phytochemical composition remains unknown, despite the use of leaves and fruits of many species in folk medicine. Due to the absence of bibliographic data, the study of their polyphenolic composition is relevant and can provide information about new plants with important medicinal applications.

Myrica faya Aiton (syn. Morella faya Ait.), commonly called "fire tree", is one of the plants associated with Laurisilva. M. faya is a species of Myrica, belonging to the genus Myrica in the family Myricaceae, native to Macaronesia (the Azores and Madeira Archipelagos and the Canary islands). It is a common evergreen shrub or small tree that usually grows around $8 \mathrm{~m}$ tall. Leaves are coriaceous, oblanceolate, $4-11 \mathrm{~cm}$ long, 1-2.5 $\mathrm{cm}$ wide; they are dark green, shiny, smooth, aromatic, and alternate along the stem. Fruits are small, red to purple when ripe, and are edible. They can be directly consumed, although they have very low sugar contents and present a bitter taste. ${ }^{4}$ Eaten raw, the berries have some astringency that limits their palatability. As a result, they are underutilized, and they are mainly used to produce jams and liquors and to add color to homemade wine. The waxy fruits were also used in the Canary Islands for skin care. ${ }^{5}$ M. faya grows abundantly in Hawaii, where it was introduced by Portuguese immigrants from Madeira and Azores in the XIX century. There, the tree is considered an invasive species, since it competes vigorously with Hawaiian native trees by its nitrogen-fixing capacity in the poor volcanic soils. In the European islands it is considered a valuable species while in Hawaii all efforts are made to eradicate it since no use is found for it. Therefore, it is important to find valuable applications for M. faya, especially taking into account that it is a protected species in Madeira Archipelago, and new applications for this plant would result in a higher concern for its current situation.

Studies on the chemical composition and antioxidant capacity of Myrica species have usually focused on Myrica rubra due to its economic importance in Asia, mainly in China. ${ }^{6-13}$ Its polyphenolic composition has been determined by HPLC-DAD-ESI-MS ${ }^{n}$ methods; $;^{7,8,11,12}$ its radical scavenging

Received: May 15, 2014

Revised: September 20, 2014

Accepted: September 22, 2014

Published: September 22, 2014 
capacity has been studied using different assays, ${ }^{6,9}$ and high amounts of phenolic compounds and high antioxidant activities were observed. In addition, research on other Myrica species has been performed. Myrica esculenta (syn. Myrica nagi) has also been reported to be rich in antioxidant compounds and to present several medicinal applications and satisfactory antioxidant and anticancer activities. ${ }^{14-16}$ However, no studies have been published regarding the chemical composition or antioxidant capacity of Myrica faya. Considering the high antioxidant activity reported in previous studies regarding other Myrica species, special attention should be paid to the chemical composition of $M$. faya and other underutilized plants.

In this work we present, for the first time, a report on the phytochemical content and antioxidant activity of Myrica faya. The methanolic extracts of its fruits and leaves were characterized by HPLC-DAD/ESI-MS ${ }^{n}$, putting special emphasis on the phenolic composition. In addition, its antioxidant capacity was evaluated using radical scavenging methods (ABTS and DPPH) and analyzing its L-ascorbic acid (L-AA) content. The obtained results were compared to the previous ones reported for other Myrica species, the main goal of this work being to find out if the chemical composition of $M$. faya makes it a valuable plant from the health and economic points of view.

\section{MATERIAL AND METHODS}

Chemicals and Instruments. All reagents and standards were of analytical reagent (AR) grade. L-Ascorbic acid (L-AA) (purity: 99\%), quercetin hydrated (99\%), potassium iodate (99\%), Folin-Ciocalteu's phenol reagent (FCR), gallic acid (99\%), rutin ( $\geq 98 \%)$, and potassium acetate $(>99.5 \%)$ were purchased from Panreac (Madrid, Spain). Ellagic acid ( $\geq 96 \%)$, 6-hydroxy-2,5,7,8-tetramethylchroman-2-carboxylic acid (Trolox) (>99.8\%), 2,2'-azinobis(3-ethylbenzthiazoline-6sulfonic acid) (ABTS) ( $\geq 99 \%$ ), and 2,2-diphenyl-1-picrylhydrazyl $(\mathrm{DPPH})(>95 \%)$ were obtained from Fluka (Lisbon, Portugal). Apigenin $(\geq 99 \%)$ and $(+)$ catechin hydrated $(>99 \%)$ were purchased from Extrasynthese (Genay, France). Caffeic acid ( $\geq 98 \%)$, potassium persulfate (99\%), sodium carbonate (p.a.), metaphosphoric acid (MPA) (33.5-36.5\%), and formic acid (98\%) were obtained from Sigma-Aldrich (St. Louis, MO); aluminum chloride hexahydrated (98\%) and potassium iodine (98\%) were from Riedel-de Haën (Hanover, Germany). Acetic acid (99.8\%), ethylenediaminetetraacetic acid disodium salt (EDTA) (98\%), and starch (98\%) were supplied by Merck (Darmstadt, Germany). Cyanidin-3-glucoside (C3G) chloride (>98\%) was obtained from Biopurify phytochemicals LTD (Chengdu, China). The methanol (99.9\%) used for the extraction of $M$. faya was purchased from Fisher (Lisbon, Portugal). LC-MS grade acetonitrile $\left(\mathrm{CH}_{3} \mathrm{CN}\right)$ (99\%) (LabScan; Dublin, Ireland) and ultrapure water (Milli-Q Waters purification system; Millipore; Milford, MA) were used for analysis.

Sample Preparation. Samples of Myrica faya were collected in the wild in Machico (Madeira Island) in July 2013 and identified by taxonomist Fátima Rocha. Voucher specimens have been stored at Madeira Botanical Garden Herbarium (Funchal, Madeira) (voucher: MADJ 13165). For analysis, plant material was separated into leaves and berries (fully ripe), destemmed, and washed. Then, samples were lyophilized to dryness (Savant vapor trap RVT400; Thermo Scientific Inc.; Waltham, MA), ground to powder, and stored at $-20{ }^{\circ} \mathrm{C}$.

Extraction of Phenolic Compounds. Before the samples were subjected to evaluation, an extraction procedure was optimized. Acetone and methanol were tested as extraction solvents. Briefly, $5 \mathrm{~g}$ of leaf powder and $100 \mathrm{~mL}$ of solvent were submitted to ultrasound sonication (Bandelin Sonorex; Germany) at $35 \mathrm{~Hz}$ and $200 \mathrm{~W}$ for 60 min (room temperature). Then, extracts were filtered and concentrated to dryness under reduced pressure in a rotary evaporator (Buchi Rotavapor R-114) at $40{ }^{\circ} \mathrm{C}$. The efficiency of the different extraction conditions was determined by means of total phenolic content assay (described below). On the basis of the results, the concentration of solvent in water $(\%, \mathrm{v} / \mathrm{v})$ and influence of extraction duration were also tested $(60,30$, and $15 \mathrm{~min})$. Finally, the optimal conditions found were applied to the target plant material, and the resulting extracts were stored at $4{ }^{\circ} \mathrm{C}$ until further analysis.

Chromatographic Conditions. The HPLC analysis was performed on a Dionex ultimate 3000 series instrument (Thermo Scientific Inc.) coupled to a binary pump, a diode-array detector (DAD), an autosampler, and a column compartment (kept at $20{ }^{\circ} \mathrm{C}$ ). Separation was achieved on a Phenomenex Gemini $\mathrm{C}_{18}$ column $(5 \mu \mathrm{m}$, $250 \mathrm{~mm} \times 3.0 \mathrm{~mm}$ i.d.) using a mobile phase composed by $\mathrm{CH}_{3} \mathrm{CN}$ (A) and water/formic acid $(0.1 \%, \mathrm{v} / \mathrm{v})$ at a flow rate of $0.4 \mathrm{~mL} \mathrm{~min}^{-1}$. The following gradient program was used: $20 \% \mathrm{~A}(0 \mathrm{~min}), 25 \% \mathrm{~A}(10$ $\mathrm{min}), 25 \% \mathrm{~A}(20 \mathrm{~min}), 50 \% \mathrm{~A}(40 \mathrm{~min}), 100 \% \mathrm{~A}(42-47 \mathrm{~min})$, and $20 \%$ A (49-55 min). Spectral data for all peaks were accumulated in the range $190-520 \mathrm{~nm}$. A solution with concentration (w/v) of $5 \mathrm{mg}$ $\mathrm{mL}^{-1}$ was prepared by dissolving the dried extract in the initial HPLC mobile phase, filtered through $0.45 \mu \mathrm{m}$ PTFE membrane filters, and $10 \mu \mathrm{L}$ was injected. The chromatographic analysis was performed in triplicate $(n=3)$ for each sample.

For HPLC-DAD/ESI-MS ${ }^{n}$ analysis, a Bruker Esquire model 6000 ion trap mass spectrometer (Bremen, Germany) with an ESI source was used. MS ${ }^{n}$ analysis worked in negative and positive mode, and scan range was set at $m / z \quad 100-1000$ with speed of $13000 \mathrm{Das}^{-1}$. The conditions of ESI were as follows: drying and nebulizer gas $\left(\mathrm{N}_{2}\right)$ flow rate and pressure, $10 \mathrm{~mL} \mathrm{~min}{ }^{-1}$ and $50 \mathrm{psi}$; capillary temperature, 325 ${ }^{\circ} \mathrm{C}$; capillary voltage, $4.5 \mathrm{keV}$; collision gas $(\mathrm{He})$ pressure and energy, $1 \times 10^{-5}$ mbar and $40 \mathrm{eV}$. The acquisition of $\mathrm{MS}^{n}$ data was made in auto $\mathrm{MS}^{n}$ mode, with isolation width of $4.0 \mathrm{~m} / z$, and fragmentation amplitude of $1.0 \mathrm{~V}\left(\mathrm{MS}^{n}\right.$ up to $\left.\mathrm{MS}^{4}\right)$. Esquire control software was used for the data acquisition and Data Analysis for processing.

Quantification of Phenolic Compounds. For this quantitative analysis, one polyphenol was selected as the standard for each group, and was used to calculate individual concentrations by HPLC-DAD. Caffeic and gallic acids were used for hydroxycinnamic and hydroxybenzoic acids, respectively. Anthocyanins standard was cyanidin 3-O-glucoside. Quercetin and apigenin were the standards used for the flavonols and flavones, respectively. (+)-Catechin hydrate and ellagic acid were used as standards for quantification of flavanols and ellagitannins. Stock standard solutions $(1000 \mathrm{mg} / \mathrm{L})$ were prepared in methanol, and calibration curves were prepared by diluting the stock solutions with the initial mobile phase. Six concentrations $(5-100 \mathrm{mg} / \mathrm{L})$ were used for the calibration, plotting peak area versus concentration, obtaining $R^{2} \geq 0.967$ in all cases. Peak area was used as the analytical signal for polyphenol quantification. Total individual phenolic contents (TIPC) was defined as the sum of the quantified phenolic compounds.

Analysis of L-AA Content and Sugars. Fresh berries were homogenized in a blender, and the $\mathrm{pH}$ was measured directly in the pulp using a Metrohm $7444 \mathrm{pH}$-meter (calibrated with standard buffer solutions of $\mathrm{pH} 7$ and $\mathrm{pH} 9$, respectively). The total soluble solids (TSS) were determined using an Atago RX-1000 refractometer, and the results were reported as Brix degrees ( ${ }^{\circ}$ Brix).

L-AA determination was carried out using the procedure indicated in our previous work. ${ }^{17}$ Briefly, $10 \mathrm{~mL}$ of extraction solution $\left(30 \mathrm{~g} \mathrm{~L}^{-1}\right.$ MPA-80 $\mathrm{mL} \mathrm{L}^{-1}$ acetic acid-1 mmol L ${ }^{-1}$ EDTA) was added to $3 \mathrm{~mL}$ of pulp, and the mixture was centrifuged (4000 rpm; $20 \mathrm{~min} ; 4{ }^{\circ} \mathrm{C}$ ). The resulting extract was immediately analyzed by iodometric titration: $1 \mathrm{~mL}$ of $10 \mathrm{~g} \mathrm{~L}^{-1}$ starch solution and $1 \mathrm{~mL}$ of $100 \mathrm{~g} \mathrm{~L}^{-1}$ potassium iodide solution were added to fruit extract (diluted 1:10 with deionized water). Then, the samples were titrated with $0.002 \mathrm{~mol}$ $\mathrm{L}^{-1}$ potassium iodate solution, until the mixture became dark blue and the color persisted for more than $60 \mathrm{~s}$. This procedure was repeated in triplicate.

TPC, TFC, and Antioxidant Capacities Assays. Total Phenolic Content (TPC). The total phenolic content was determined by the Folin-Ciocalteu method. ${ }^{18}$ Briefly, $50 \mu \mathrm{L}$ aliquots $\left(5 \mathrm{mg} \mathrm{mL}^{-1}\right.$ of dried extract dissolved in methanol) were mixed with $1.25 \mathrm{~mL}$ of FCR (diluted $1: 10$ ) and $1 \mathrm{~mL}$ of $7.5 \% \mathrm{Na}_{2} \mathrm{CO}_{3}$ solution. After $30 \mathrm{~min}$ in darkness and room temperature, the absorbance was measured at 765 
$\mathrm{nm}(n=3)$ in a PerkinElmer UV-vis Lambda 2 spectrophotometer. The amounts of total phenolics were expressed as $\mathrm{mg}$ gallic acid equivalents (GAE)/100 g of dried extract (DE).

Total Flavonoid Content (TFC). The total flavonoid content was evaluated using the aluminum chloride colorimetric method: ${ }^{18} 0.5 \mathrm{~mL}$ of methanolic extract $\left(2.5 \mathrm{mg} \mathrm{mL} \mathrm{m}^{-1}\right)$ was mixed with $1.5 \mathrm{~mL}$ of methanol, $2.8 \mathrm{~mL}$ of distilled water, $0.1 \mathrm{~mL}$ of $\mathrm{CH}_{3} \mathrm{COOK}(1 \mathrm{~mol}$ $\mathrm{L}^{-1}$ ), and $0.1 \mathrm{~mL}$ of $\mathrm{AlCl}_{3} \cdot 6 \mathrm{H}_{2} \mathrm{O}$. The absorbance was measured at 415 $\mathrm{nm}$ after $30 \mathrm{~min}$ of reaction. The final results were expressed as $\mathrm{mg}$ of rutin equivalent (RUE)/100 g DE.

ABTS Radical Scavenging Activity. The $\mathrm{ABTS}^{\bullet+}$ assay was performed according to Gouveia et al. ${ }^{18}$ For each analysis, $40 \mu \mathrm{L}$ of methanolic solution was added to $1.96 \mathrm{~mL}$ of $\mathrm{ABTS}^{\bullet+}$ solution (diluted in phosphate buffered saline, PBS; absorbance $0.700 \pm 0.021$ ). The reduction of absorbance at $734 \mathrm{~nm}$ was measured during $10 \mathrm{~min}$, and the results were expressed as $\mu \mathrm{mol}$ Trolox equivalent (TE)/100 g DE.

DPPH Radical Scavenging Activity. The DPPH assay followed a method previously reported: ${ }^{18} 100 \mu \mathrm{L}$ of methanolic solution $(5 \mathrm{mg}$ $\mathrm{mL}^{-1}$ ) were added to $3.5 \mathrm{~mL}$ of DPPH radical solution $(0.06 \mathrm{~mol}$ $\left.\mathrm{L}^{-1}\right)$. The decrease in absorbance at $516 \mathrm{~nm}$ was measured every minute during $30 \mathrm{~min}$. The DPPH results were expressed as $\mu \mathrm{mol}$ Trolox equivalent (TE)/100 g DE.

Statistical Analysis. Analysis of variance (ANOVA) was used to evaluate the results obtained in L-AA, TPC, TFC, and antioxidant assays determinations (IBM SPSS Statistics 20, SPSS, Inc.).

\section{RESULTS AND DISCUSSION}

In this study, we aimed to establish, for the first time, the phenolic profile from different morphological parts of Myrica faya. Prior to the phenolic characterization, the influence of different experimental variables on the extraction procedure (solvent type, concentration, and duration of extraction) was investigated to increase the extraction efficiency of phenolics.

The results from the extraction experiments are shown in Figure 1.

A significant difference $(p<0.05)$ was found between extraction with pure methanol or pure acetone, with a higher extraction yield using pure methanol (Figure 1a). Our results contradict those of Saini et al., who reported that acetone was more efficient than methanol for the extraction of phenolics from M. esculenta. ${ }^{16}$ On the basis of our data, methanol was chosen for further investigations, and results showed that an increase in the percentage of this solvent influenced positively the extraction efficiency (Figure $1 \mathrm{~b}$ ). Significant differences $(p$ $<0.05$ ) were observed between the different concentrations of methanol, except for $90 \%$ and $80 \%$. Moreover, the yields of phenolic content were equal $(p>0.05)$ when using aqueous methanol (80\%) and acetone as the extraction solvents. Taking this fact into account, pure methanol was used to evaluate the influence of extraction time, and the results indicated that increasing the extraction duration had a positive effect on the extraction efficiency $(p<0.05)$ (Figure 1c). Thus, an extraction time of $60 \mathrm{~min}$ with $100 \%$ methanol was considered as optimum.

HPLC-DAD-ESI/MS ${ }^{n}$ Screening. Figure 2 shows the chromatogram obtained during the analysis of the methanolic extracts from Myrica faya by HPLC-DAD-ESI/MS ${ }^{n}$. The identification of compounds was carried out by comparison of their UV-vis spectra and mass spectrometric data obtained under negative electrospray ionization $\left(\mathrm{ESI}^{-}\right)$conditions with the data available in scientific literature.

The method achieved a good separation, and no relevant variation was observed in the three determinations performed for each sample. In general, in the $\mathrm{MS}^{1}$ spectrum the most
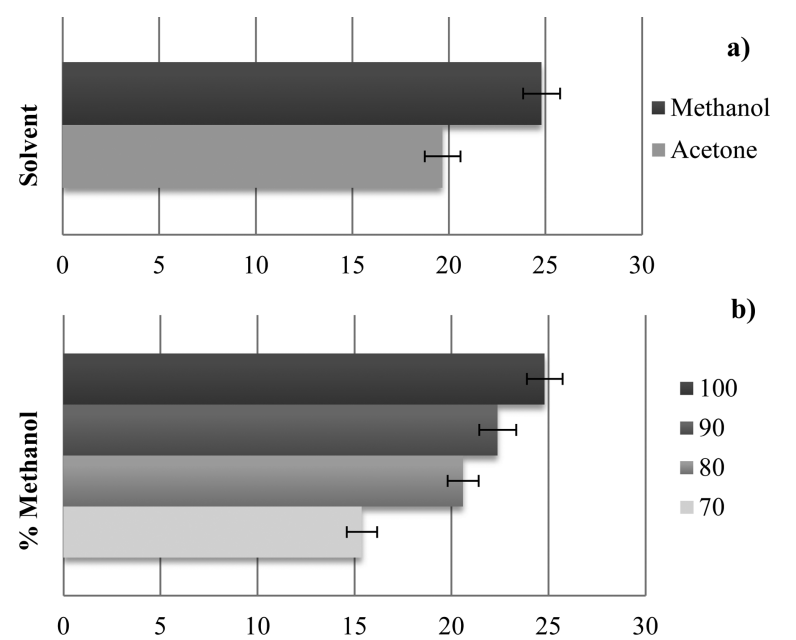

b)

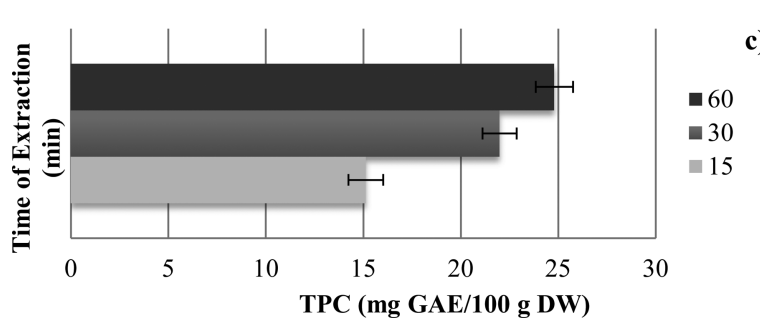

Figure 1. Extraction efficiency of different extraction conditions determined by TPC (mg/100 g DW) in Myrica faya leaves: (a) effect of solvent (methanol versus acetone); (b) effect of methanol concentration (v/v); (c) effect of extraction time. All extractions procedures were repeated three times $(n=3)$.

intense peak corresponded to the deprotonated molecular ion $[\mathrm{M}-\mathrm{H}]^{-}$. The mass spectra of the conjugated form of the phenolic compounds showed the aglycone ion as a result of the loss of moieties like hexosyl, deoxyhexosyl, pentosyl, rutinosyl, caffeoyl, and glucuronyl $(-162,-146,-132,-308,-162$, and $-176 \mathrm{Da}$, respectively). The identification of the compounds detected in leaf and berries extracts is presented in Tables 1 and 2 , respectively, and their chemical structures are shown in Figure 3.

Compounds were numbered by their elution order, since most of them were not found in both samples (leaves and berries). More than 50 different compounds were detected and classified into two main groups: flavonoids (flavan-3-ols, flavones, isoflavones, and flavonols) and phenolic acids (hydroxybenzoic and hydroxycinnamic acids). Quinic acid and derivatives were also relevant in leaves. Additionally, mass spectra data from the positive ionization mode $\left(\mathrm{ESI}^{+}\right)$was used for confirmation of the anthocyanidin compounds, namely cyanidin-3-glucoside and delphinidin-O-hexoside, in berries. A characteristic esterification with gallic acid was found in the majority of the compounds, representing the dominant group bound to polyphenols of leaves and berries.

The phenolic profiles obtained by our HPLC-UV/DAD-MS ${ }^{n}$ analysis were similar to previous reports on Myrica. ${ }^{6-8,10-13,15}$ In addition, we were still able to identify for the first time in this genus 26 compounds, namely flavones, ellagitannins, lignans, terpenoids, among others. The analysis showed that leaves of M. faya were significantly more complex when compared to berries, most of the identified compounds exclusively being detected in the leaf extracts. Nevertheless, some compounds were only detected in berries $(2,4,6,9,13,16,19,24$, and 26). 




Figure 2. HPLC-DAD-ESI/MS ${ }^{n}$ base peak chromatograms (BPC) of the methanolic extracts from Myrica faya: leaves and berries.

Negative Mode lonization. For the analysis of the phenolic composition of $M$. faya, both the positive and negative ionization modes were used. However, the majority of the information was obtained using the negative mode, and the positive mode was mainly used for confirmation purposes.

Identification of Phenolic Acids. Compound 4 presented $[\mathrm{M}-\mathrm{H}]^{-}$ion at $m / z 341$. It suffered the neutral loss of $162 \mathrm{Da}$ (hexoside), producing a fragment ion at $\mathrm{m} / z$ 179. This ion suffered further fragmentation, producing fragment ions at $\mathrm{m} / z$ 161 and 135, which are typical from caffeic acid, so the compound was identified as caffeic acid $O$-hexoside. ${ }^{19}$

Compound 29 exhibited a $[\mathrm{M}-\mathrm{H}]^{-}$ion at $m / z 415$ and was characterized as a caffeic acid derivative. Its $\mathrm{MS}^{n}$ spectrum was identical to that described previously in $\mathrm{H}_{\text {. obconicum }}{ }^{19}$ by our group. To our best knowledge, the presence of caffeic acid derivatives has not been reported, so far, in Myrica.

Compound 17 displayed a $[\mathrm{M}-\mathrm{H}]^{-}$ion at $\mathrm{m} / z$ 421, which gave origin to an ion at $m / z 385$ (by loss of $36 \mathrm{Da}$ ). Further fragmentation led to sinapic acid aglycone at $m / z 223$ (by loss of $162 \mathrm{Da}),{ }^{10}$ being characterized as sinapic acid-O-hexoside derivative.

Compounds 37 and 39, showing $[\mathrm{M}-\mathrm{H}]^{-}$ions at $m / z 511$ and 481, were identified, for the first time in Myrica, as derivatives of sinapic acid- $O$-hexoside and ferulic acid- $O$ hexoside, respectively. Both showed identical neutral losses at $\mathrm{MS}^{2}(126+162 \mathrm{Da})$, but the presence of sinapic and ferulic acids led to different characterizations. While the $162 \mathrm{Da}$ loss are attributed to hexoside units attached to the aglycones, the $126 \mathrm{Da}$ loss could not be identified.
Compound 6 with $[\mathrm{M}-\mathrm{H}]^{-}$at $m / z 331$ was plausibly identified as galloyl-O-hexoside, according to previous studies in pomegranate. ${ }^{20,21}$

Compound 8 exhibited $[\mathrm{M}-\mathrm{H}]^{-}$ion at $\mathrm{m} / z 467$ and fragmented into ion at $m / z 169$ [gallic acid $-\mathrm{H}]^{-}$due to loss of $298 \mathrm{Da}$. In the absence of more specific data, 8 was assigned as a gallic acid derivative.

Compound 18, with $[\mathrm{M}-\mathrm{H}]^{-}$at $m / z 285$, was identified as protocatechuic acid-O-pentoside based on bibliographic data. ${ }^{22}$ The presence of this hydroxybenzoic acid in Myrica species is consistent with previous reports. ${ }^{13}$

Flavonoids. In this study, flavonoids (flavones, flavonols, and flavan-3-ols) were detected in their glycosylated form and/or esterified with acyl groups and were the most abundant components identified.

Compound 11 had an $[\mathrm{M}-\mathrm{H}]^{-}$ion at $m / z 761$ and displayed typical product ions for galloyl-di(epi)gallocatechin at $m / z 609[\mathrm{M}-152-\mathrm{H}]^{-}, 591[\mathrm{M}-170-\mathrm{H}]^{-}$, and $423[\mathrm{M}$ $-170-168-\mathrm{H}]^{-}$, which corresponded to losses of galloyl moieties (170 and $152 \mathrm{Da}$ ) and retro-Diels-Alder reaction product ion $(168 \mathrm{Da})$, respectively. This fragmentation behavior is congruent with the previously published ${ }^{11}$ for this compound in Myrica rubra.

Compound 12 was assigned as gallo(epi)catechin with characteristic $[\mathrm{M}-\mathrm{H}]^{-}$ion at $\mathrm{m} / z 305$, based on previous characterization on pomegranate. $^{21}$

Compound 13 displayed $[\mathrm{M}-\mathrm{H}]^{-}$ion at $m / z 483$ and gave origin to a product ion at $\mathrm{m} / z 447$ (by loss of $36 \mathrm{Da}$ ). Sequential loss of a hexoside moiety produced luteolin aglycone 
Table 1. Characterization of Phenolic and Organic Compounds of the Methanolic Extracts of Leaves from Myrica faya

\begin{tabular}{|c|c|c|c|c|c|}
\hline no. & $t_{\mathrm{R}}(\min )$ & $\lambda_{\max }^{a}(\mathrm{~nm})$ & {$[\mathrm{M}-\mathrm{H}]^{-}(m / z)$} & HPLC-DAD-ESI $/ \mathrm{MS}^{n} m / z$ (\% base peak) & assigned identity \\
\hline 1 & 3.0 & 234,273 & $683[2 \mathrm{M}-\mathrm{H}]^{-}$ & $\begin{array}{l}\mathrm{MS}^{2}[683]: 341(100), 342(10.3) \\
\operatorname{MS}^{3}[683 \rightarrow 341]: 179(100), 161(24.1), 143(17.7), 119(15.5), \\
\quad 113(18.0) \\
\operatorname{MS}^{4}[683 \rightarrow 341 \rightarrow 179]: 161(29.7), 149(22.7), 143(87.7), 113(48.8), \\
\quad 101(30.1), 89(100)\end{array}$ & unknown \\
\hline 3 & 3.1 & & 533 & $\begin{array}{l}\mathrm{MS}^{2}[533]: 191(100) \\
\mathrm{MS}^{3}[533 \rightarrow 191]: 173(100), 127(64.9), 109(32.8), 99(50.1) \\
\quad 93(59.0), 85(42.1) \\
\mathrm{MS}^{4}[533 \rightarrow 191 \rightarrow 173]: 109(100)\end{array}$ & quinic acid derivative \\
\hline 5 & 3.3 & & 191 & $\begin{array}{l}\operatorname{MS}^{2}[191]: 173(58.2), 127(100), 111(40.5), 109(23.8), 93(41.7), \\
85(37.1), 109(23.8) \\
\operatorname{MS}^{3}[191 \rightarrow 127]: 109(100), 99(53.9), 85(39.8)\end{array}$ & quinic acid \\
\hline 7 & 3.8 & & $383[2 \mathrm{M}-\mathrm{H}]^{-}$ & $\begin{array}{l}\mathrm{MS}^{2}[383]: 191(100) \\
\mathrm{MS}^{3}[383 \rightarrow 191]: 127(100), 85(69.8), 93(58.4), 109(60.4) \\
\quad 111(43.0), 173(24.5)\end{array}$ & quinic acid dimer \\
\hline 8 & 4.3 & 213,273 & 467 & $\begin{array}{l}\mathrm{MS}^{2}[467]: 436(36.6), 391(52.4), 301(42.1), 275(71.9), 169(100) \\
\mathrm{MS}^{3}[467 \rightarrow 169]: 125(100), 123(41.5)\end{array}$ & gallic acid derivative \\
\hline 11 & 4.7 & 207,276 & 761 & $\begin{array}{l}\mathrm{MS}^{2}[761]: 635(17.8), 609(69.0), 575(36.6), 591(51.2), 593(33.8), \\
\quad 423(100), 305(38.4) \\
\mathrm{MS}^{3}[761 \rightarrow 423]: 305(51.2), 297(61.7), 283(100), 255(77.4), \\
\quad 243(36.5) \\
\mathrm{MS}^{4}[761 \rightarrow 423 \rightarrow 283]: 255(33.5), 241(100)\end{array}$ & galloyl(epi)gallocatechin dimer \\
\hline 12 & 4.8 & & 305 & $\begin{array}{l}\mathrm{MS}^{2}[305]: 261(54.6), 221(34.0), 219(85.6), 204(21.7), 179(100) \\
\quad 166(17.0), 139(16.5), 137(63.1) \\
\mathrm{MS}^{3}[305 \rightarrow 179]: 163(100), 152(45.5), 151(77.3), 135(32.1)\end{array}$ & gallo(epi) catechin ${ }^{b}$ \\
\hline 14 & 5.0 & & 935 & $\begin{array}{l}\operatorname{MS}^{2}[935]: 917(20.9), 659(21.2), 633(100), 615(36.7), 571(18.5), \\
329(25.4), 301(21.9), 299(49.4) \\
\operatorname{MS}^{3}[935 \rightarrow 633]: 615(76.5), 571(100), 481(44.3), 383(31.7) \\
329(76.1), 301(28.7), 299(97.9), 275(26.5)\end{array}$ & $\begin{array}{l}\text { galloyl-bis-HHDP-O-hexoside } \\
\quad(\text { Casuarinin })^{b}\end{array}$ \\
\hline 15 & 5.3 & & 447 & $\begin{array}{l}\operatorname{MS}^{2}[447]: 401(100) \\
\operatorname{MS}^{3}[447 \rightarrow 401]: 269(100), 179(48.2), 161(38.9), 159(14.7) \\
\operatorname{MS}^{4}[447 \rightarrow 401 \rightarrow 269]: 161(100), 143(32.5), 99(17.5)\end{array}$ & $\begin{array}{l}\text { benzyl alcohol hexose pentose } \\
\text { (formate adduct) })^{b}\end{array}$ \\
\hline 17 & 5.6 & & 421 & $\begin{array}{l}\mathrm{MS}^{2}[421]: 386(68.4), 385(100), 305(12), 205(90.2), 153(14.1) \\
\operatorname{MS}^{3}[421 \rightarrow 385]: 326(22.4), 265(23.5), 224(13.6), 223(100) \\
\quad 205(23.1) \\
\operatorname{MS}^{4}[421 \rightarrow 385 \rightarrow 223]: 208(46.4), 179(79), 164(100)\end{array}$ & sinapic acid-O-hexoside derivative \\
\hline 18 & 5.9 & 209,277 & 285 & $\begin{array}{l}\operatorname{MS}^{2}[285]: 154(11.8), 153(100), 152(21.2), 109(12.0) \\
M^{3}[285 \rightarrow 153]: 109(77.0), 108(100)\end{array}$ & protocathechuic acid-O-pentoside \\
\hline 20 & 6.8 & & 457 & $\begin{array}{l}\mathrm{MS}^{2}[457]: 331(19.8), 319(14.8), 305(12.4), 193(16.3), 169(100) \\
\operatorname{MS}^{3}[457 \rightarrow 169]: 125(100)\end{array}$ & gallo(epi)catechin-O-gallate \\
\hline 21 & 7.0 & 209,275 & $915[2 \mathrm{M}-\mathrm{H}]^{-}$ & $\begin{array}{l}\mathrm{MS}^{2}[915]: 458(14.6), 457(100) \\
\mathrm{MS}^{2}[915 \rightarrow 457]: 331(27.4), 305(33.1), 193(16.2), 169(100) \\
\mathrm{MS}^{3}[915 \rightarrow 457 \rightarrow 169]: 125(100)\end{array}$ & gallo(epi)catechin-O-gallatedimer \\
\hline 22 & 7.5 & $\begin{array}{l}209,268 \\
359\end{array}$ & 479 & $\begin{array}{l}\operatorname{MS}^{2}[479]: 317(100), 316(92.3), 179(16.0) \\
\operatorname{MS}^{3}[479 \rightarrow 317]: 287(30.6), 271(81.6), 193(40.6), 179(100)\end{array}$ & myricetin- $O$-hexoside \\
\hline 23 & 7.7 & & 631 & $\begin{array}{l}\operatorname{MS}^{2}[631]: 479(39.7), 318(12.7), 317(100) \\
\operatorname{MS}^{3}[631 \rightarrow 317]: 179(100), 151(39.1)\end{array}$ & myricetin- $O$-(O-galloyl $)$ hexoside \\
\hline 25 & 9.7 & $\begin{array}{l}209,262 \\
349\end{array}$ & 463 & $\begin{array}{l}\operatorname{MS}^{2}[463]: 318(10.1), 317(100), 316(64.5) \\
\operatorname{MS}^{3}[463 \rightarrow 317]: 288(11.4), 287(24.7), 272(27.2), 271(59.7) \\
\quad 270(35.9), 179(100), 151(10.7)\end{array}$ & myricetin-O-deoxyhexoside \\
\hline 27 & 10.3 & 207,358 & 615 & $\begin{array}{l}\operatorname{MS}^{2}[615]: 302(14.7), 301(100), 313(16.6), 463 \\
\operatorname{MS}^{3}[615 \rightarrow 301]: 179(100), 193(15.5), 151(63.4) \\
\operatorname{MS}^{4}[615 \rightarrow 301 \rightarrow 179]: 257(11.5), 151(100), 169(64.3)\end{array}$ & galloylquercetin- $O$ - hexoside ${ }^{b}$ \\
\hline 28 & 11.0 & & 593 & $\begin{array}{l}\mathrm{MS}^{2}[593]: 285(100), 286(18.8) \\
\mathrm{MS}^{3}[593 \rightarrow 285]: 257(100), 241(58.8), 229(35.9), 197(17.6) \\
\quad 169(23.2), 163(39.6), 93(30.3) \\
\mathrm{MS}^{4}[593 \rightarrow 285 \rightarrow 257]: 255(22.1), 151(100)\end{array}$ & kaempferol-O-rutinoside ${ }^{b}$ \\
\hline 29 & 11.6 & & 415 & $\begin{array}{l}\operatorname{MS}^{2}[415]: 369(65.9), 225(30.0), 179(100), 161(11.4), 149(10.7) \\
\quad 143(12.2) \\
\operatorname{MS}^{3}[415 \rightarrow 179]: 161(100), 135(48.4), 89(49.6)\end{array}$ & $\begin{array}{l}\text { caffeic acid derivative (formate } \\
\text { adduct) }\end{array}$ \\
\hline 30 & 12.1 & $\begin{array}{l}208,267 \\
343\end{array}$ & 447 & $\mathrm{MS}^{2}[447]: 285$ (91.8), $284(100), 255$ (22.0), $256(16.8)$ & kaempferol-O-hexoside ${ }^{b}$ \\
\hline
\end{tabular}




\section{Table 1. continued}

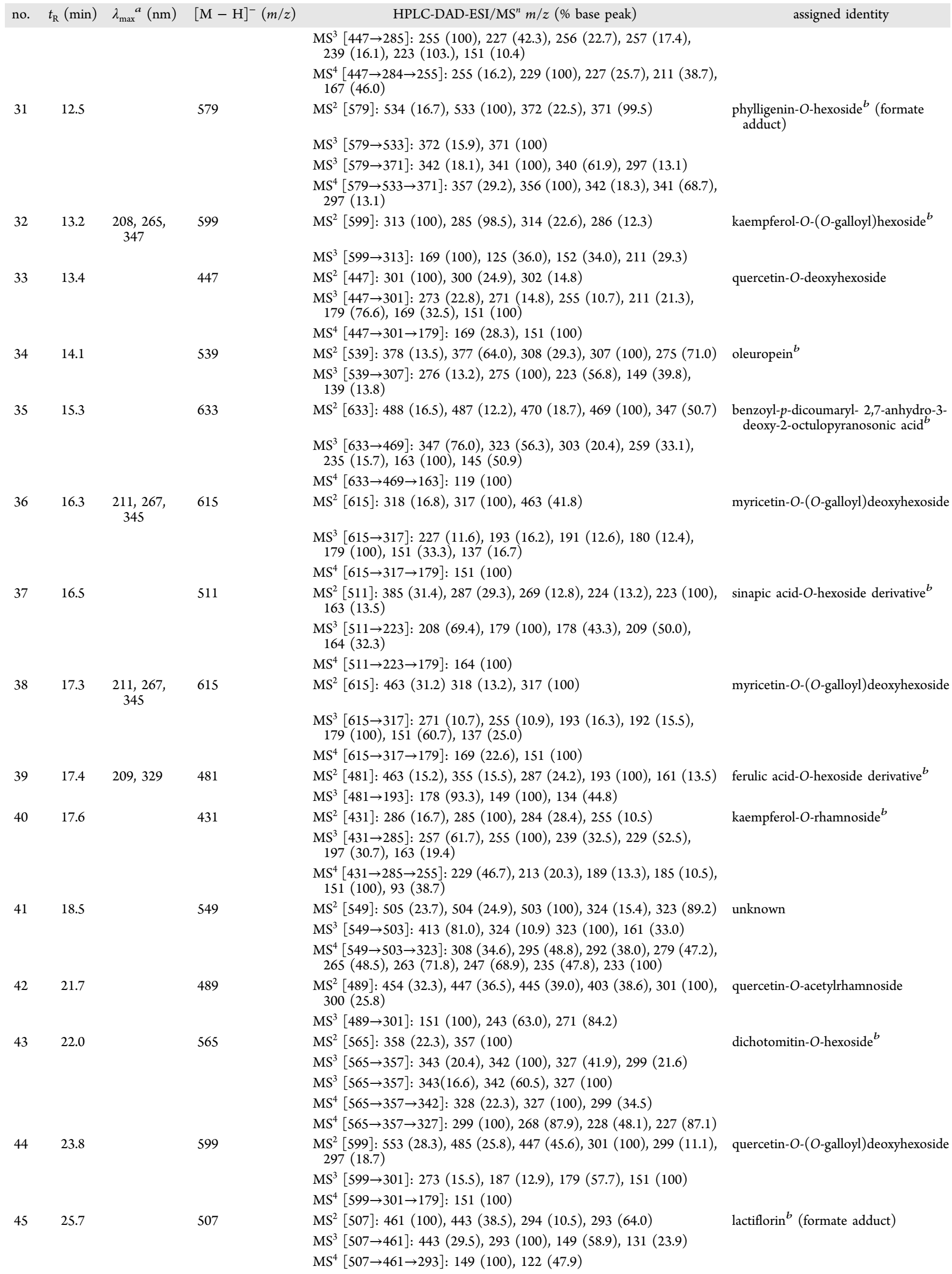




\section{Table 1. continued}

\begin{tabular}{|c|c|c|c|c|}
\hline no. & $t_{\mathrm{R}}(\min ) \quad \lambda_{\max }{ }^{a}(\mathrm{~nm})$ & {$[\mathrm{M}-\mathrm{H}]^{-}(m / z)$} & HPLC-DAD-ESI/MS ${ }^{n} m / z$ (\% base peak) & assigned identity \\
\hline 46 & 26.6 & 491 & $\begin{array}{l}\operatorname{MS}^{2}[491]: 371(12.9), 330(28.5), 330(20.3), 329(100), 314(10.7) \\
\operatorname{MS}^{3}[491 \rightarrow 329]: 315(53.0), 314(100) \\
\operatorname{MS}^{3}[491 \rightarrow 329 \rightarrow 314]: 300(40.3), 299(100)\end{array}$ & tricin-O-hexoside ${ }^{b}$ \\
\hline 47 & 27.3 & 491 & $\begin{array}{l}\operatorname{MS}^{2}[491]: 330(26.9), 329(100) \\
\operatorname{MS}^{3}[491 \rightarrow 329]: 315(14.3), 314(100), 136(38.6), 135(40.1) \\
\text { MS }^{4}[491 \rightarrow 329 \rightarrow 314]: 300(43.1), 299(100)\end{array}$ & tricin-O-hexoside ${ }^{b}$ \\
\hline 48 & 27.8 & 555 & $\begin{array}{l}\operatorname{MS}^{2}[555]: 417(21.7), 305(32.9), 287(27.1), 269(100), 267(22.4), \\
223(54.3), 161(22.2) \\
\operatorname{MS}^{3}[555 \rightarrow 269]: 251(20.5), 241(38.8), 227(45.9), 226(51.3), \\
225(32.3), 223(100), 197(48.9), 195(33.5), 179(17.1) \\
\mathrm{MS}^{4}[555 \rightarrow 269 \rightarrow 223]: 197(100)\end{array}$ & baicalein derivative $^{b}$ \\
\hline 49 & 29.1 & 491 & $\begin{array}{l}\mathrm{MS}^{2}[491]: 473(20.8), 330(15.2), 329(100) \\
\mathrm{MS}^{3}[491 \rightarrow 329]: 314(100), 299(70.5), 271(52.1), 193(48.3) \\
\quad 181(62.0), 135(51.6)\end{array}$ & tricin-O-hexoside ${ }^{b}$ \\
\hline 50 & 29.4 & 563 & $\begin{array}{l}\mathrm{MS}^{2}[563]: 356(11.0), 355(100) \\
\mathrm{MS}^{3}[563 \rightarrow 355]: 341(12.8), 340(100), 325(43.4) \\
\mathrm{MS}^{4}[563 \rightarrow 355 \rightarrow 340]: 326(15.2), 325(100), 296(79.8), 281(36.7), \\
\quad 212(27.6) \\
\mathrm{MS}^{4}[563 \rightarrow 355 \rightarrow 325]: 296(100), 281(79.8), 212(27.6)\end{array}$ & conidendrin-O-hexoside ${ }^{b}$ \\
\hline 51 & 29.8 & 583 & $\begin{array}{l}\operatorname{MS}^{2}[583]: 286(17.7), 285(100) \\
\operatorname{MS}^{3}[583 \rightarrow 285]: 267(55.6), 257(100), 241(43.8), 151 \text { (93.5), } \\
\quad 169(48.8)\end{array}$ & kaempferol derivative \\
\hline 52 & 30.4 & 535 & $\begin{array}{l}\operatorname{MS}^{2}[535]: 490(31.6), 489(100) \\
\operatorname{MS}^{3}[535 \rightarrow 489]: 327(46.0), 313(100), 298(55.5), 283(33.6) \\
\operatorname{MS}^{4}[535 \rightarrow 489 \rightarrow 313]: 298(100), 283(22.7), 269(32.4), 254(29.4)\end{array}$ & $\begin{array}{l}\text { 5,7-dihydroxy-6,8-dimethoxyflavone- } \\
\text { 7-O-glucuronide }{ }^{b}\end{array}$ \\
\hline 53 & 30.9 & 677 & $\begin{array}{l}\operatorname{MS}^{2}[677]: 593(75.0), 575(61.3), 285(100), 284(99.2), 268(17.7) \\
255(22.3), 229(19.3) \\
\operatorname{MS}^{3}[677 \rightarrow 285]: 283(51.6), 257(100), 255(65.6), 241(74.3) \\
229(38.5), 197(49.1)\end{array}$ & $\begin{array}{l}\text { kaempferol-O-rutinoside } \\
\text { derivative }^{b}\end{array}$ \\
\hline 54 & 31.9 & 779 & $\begin{array}{l}\operatorname{MS}^{2}[779]: 634(26.5), 633(100), 616(26.2), 615(74.3), 469(38.7) \\
\operatorname{MS}^{3}[779 \rightarrow 633]: 488(18.7), 487(17.9), 470(35.0), 469(100) \\
\quad 325(17.3), 265(11.1) \\
\mathrm{MS}^{4}[779 \rightarrow 633 \rightarrow 469]: 307(88.9), 163(100), 145(49.5)\end{array}$ & $\begin{array}{l}\text { benzoyl-p-tricoumaryl- 2,7-anhydro-3- } \\
\text { deoxy-2-octulopyranosonic acid }{ }^{b}\end{array}$ \\
\hline 55 & 32.8 & 695 & $\begin{array}{l}\mathrm{MS}^{2}[695]: 488(25.6), 487(100) \\
\operatorname{MS}^{3}[695 \rightarrow 487]: 421(31.1), 410(48.3), 409(100), 401(35.3), \\
\quad 391(59.4), 390(16.2) \\
\mathrm{MS}^{4}[695 \rightarrow 487 \rightarrow 409]: 392(15.7), 391(35.2), 380(34.7), 379(100), \\
\quad 377(25.2), 359(24.2)\end{array}$ & unknown \\
\hline 56 & 33.6 & 673 & $\begin{array}{l}\operatorname{MS}^{2}[673]: 638(23.4), 637(100), 655(22.6), 619(15.2), 611(15.7), \\
\quad 595(16.7) \\
\operatorname{MS}^{3}[673 \rightarrow 637]: 609(54.4), 401(26.0), 365(37.7), 332(28.9) \\
\quad 209(100), 203(64.2)\end{array}$ & unknown \\
\hline 58 & 34.8 & 515 & $\begin{array}{l}\operatorname{MS}^{2}[515]: 454(55.9), 269(100), 243(18.2), 241(28.1), 227(71.6) \\
\quad 183(40.5) \\
\operatorname{MS}^{3}[515 \rightarrow 269]: 228(100), 213(80.2), 149(14.8)\end{array}$ & unknown \\
\hline 59 & 38.5 & 515 & $\begin{array}{l}\operatorname{MS}^{2}[515]: 285(43.9), 284(100), 255(23.0) \\
\operatorname{MS}^{3}[515 \rightarrow 284]: 257(19.2), 256(25.0), 255(100), 242(34.0), \\
\quad 195(21.1)\end{array}$ & kaempferol derivative \\
\hline
\end{tabular}

${ }^{a}$ Wavelengths not provided when the UV spectrum was not properly observed due to low intensity. ${ }^{b}$ Compound identified for the first time in Myrica genus.

$(\mathrm{m} / z$ 285). Thus, 13 was identified as a luteolin-O-hexoside derivative, reported for the first time in Myrica.

(epi)Catechin monomer and (epi)catechin-O-gallate (compounds 19 and 26) displayed $[\mathrm{M}-\mathrm{H}]^{-}$ions at $\mathrm{m} / \mathrm{z} 289$ and 441 , respectively, and were assigned according to previous characterizations $^{23}$ in grape pomace. Catechin has been previously detected in $M$. esculenta by an HPLC-PDA method. ${ }^{15}$

Compound 20 exhibited a $[\mathrm{M}-\mathrm{H}]^{-}$ion at $m / z 457$ and was assigned as gallocatechin-O-gallate, on the basis of previous studies in M. rubra. ${ }^{11}$ Although compound 20 was detected before in leaves of bayberry, we report here for the first time its presence in fruits of this genus. With $[\mathrm{M}-\mathrm{H}]^{-}$ion at $m / z 915$ and $\mathrm{MS}^{2}$ product ion at $m / z 457$, compound 21 was plausibly identified as a dimer of gallo(epi)catechin- $O$-gallate.

Conjugates of myricetin (compounds 22, 23, 25, 36, and 38), quercetin (compounds 33, 42, and 44), and kaempferol (compounds 28, 30, 32, and 40) were characterized according to the sugar moieties attached to their aglycones (at $m / z 317$, 301 , and 285, respectively). The characterizations of these compounds in Myrica faya were supported by previous reports in $M$. rubra ${ }^{6-8,11,13}$ and pomegranate. ${ }^{20}$ Myricetin derivatives were reported as the major flavonoids in extracts of M. rubra's leaves and berries. Besides kaempferol-O-hexoside (30), all 
Table 2. Characterization of Phenolic and Organic Compounds of the Methanolic Extracts of Berries from Myrica faya

\begin{tabular}{|c|c|c|c|c|c|}
\hline no. & $t_{\mathrm{R}}(\min )$ & $\lambda_{\max }^{a}(\mathrm{~nm})$ & {$[\mathrm{M}-\mathrm{H}]^{-}(m / z)$} & HPLC-DAD-ESI $/ \mathrm{MS}^{n} m / z$ ( $\%$ base peak) & assigned identity \\
\hline 2 & 3.0 & $\begin{array}{l}219,280 \\
516\end{array}$ & $449(+)$ & $\begin{array}{l}\mathrm{MS}^{2}[449]: 288(14.1), 287(100) \\
\mathrm{MS}^{3}[449 \rightarrow 287]: 241(87.2), 213(100), 193(58.2), 185(47.6) \\
\quad 175(57.5), 169(50.1) 165(24.1), 161(31.3), 137(69.0) \\
\mathrm{MS}^{4}[449 \rightarrow 287 \rightarrow 213]: 167(100)\end{array}$ & cyanidin-3-glucoside \\
\hline 4 & 3.2 & 234,274 & 341 & $\begin{array}{l}\mathrm{MS}^{2}[341]: 179(100), 135(22.7) \\
\mathrm{MS}^{3}[341 \rightarrow 179]: 161(11.7), 135(100)\end{array}$ & caffeic acid-O-hexoside ${ }^{b}$ \\
\hline 5 & 3.3 & & 191 & $\begin{array}{l}\mathrm{MS}^{2}[191]: 173(58.2), 127(100), 111(40.5), 109(23.8), 93(41.7) \\
\quad 85(37.1), 109(23.8) \\
\operatorname{MS}^{3}[191 \rightarrow 127]: 109(100), 99(53.9), 85(39.8)\end{array}$ & quinic acid \\
\hline 6 & 3.4 & & 331 & $\begin{array}{l}\mathrm{MS}^{2}[331]: 271(30.5), 169(100), 125(29.7) \\
\mathrm{MS}^{3}[331 \rightarrow 169]: 125(100)\end{array}$ & galloyl-O-hexoside $^{b}$ \\
\hline 9 & 4.3 & 280,519 & $465(+)$ & $\begin{array}{l}\mathrm{MS}^{2}[465]: 304(17.7), 303(100) \\
\mathrm{MS}^{3}[465 \rightarrow 303]: 258(22.3), 257(100), 247(11.8), 229(80.5) \\
\quad 163(10.3), 135(31.4) \\
\mathrm{MS}^{4}[465 \rightarrow 303 \rightarrow 257]: 229(56.4), 213(100), 173(63.4)\end{array}$ & delphinidin-O-hexoside ${ }^{b}$ \\
\hline 10 & 4.5 & & 405 & $\begin{array}{l}\mathrm{MS}^{2}[405]: 388(11.6), 387(16.7), 191(100) \\
\mathrm{MS}^{3}[405 \rightarrow 191]: 173(23.3), 127(100), 111(14.0), 93(10.2)\end{array}$ & quinic acid derivative \\
\hline 13 & 4.8 & & 483 & $\begin{array}{l}\mathrm{MS}^{2}[483]: 448(20.6), 447(100) \\
\mathrm{MS}^{3}[483 \rightarrow 447]: 286(17.8), 285(100), 284(20.5) \\
\mathrm{MS}^{4}[483 \rightarrow 447 \rightarrow 285]: 243(100), 241(55.0), 217(29.2), 199(16.8) \\
\quad, 167(41.9), 125(37.7)\end{array}$ & luteolin-O-hexoside derivative ${ }^{b}$ \\
\hline 16 & 5.4 & & 431 & $\begin{array}{l}\mathrm{MS}^{2}[431]: 386(41.1), 385(100), 384(22.1), 175(17.4) \\
\mathrm{MS}^{3}[431 \rightarrow 385]: 223(100), 153(30.1), 138(11.4) \\
\mathrm{MS}^{4}[431 \rightarrow 385 \rightarrow 223]: 206(47.0), 153(100), 147(39.9)\end{array}$ & roseoside $^{b}$ (formate adduct) \\
\hline 19 & 6.4 & & 289 & $\begin{array}{l}\mathrm{MS}^{2}[289]: 247(19.3), 245(100), 205(48.0), 203(16.2), 179(14.6) \\
\quad 161(15.7) \\
\mathrm{MS}^{3}[289 \rightarrow 245]: 205(40.1), 204(85.6), 203(100), 202(47.2) \\
\quad 187(53.4), 162(17.2), 121(56.3)\end{array}$ & (epi) catechin $^{b}$ \\
\hline 20 & 6.8 & & 457 & $\begin{array}{l}\mathrm{MS}^{2}[457]: 331(19.8), 319(14.8), 305(12.4), 193(16.3), 169(100) \\
\mathrm{MS}^{3}[457 \rightarrow 169]: 125(100)\end{array}$ & gallo(epi)catechin-O-gallate ${ }^{b}$ \\
\hline 22 & 7.5 & $\begin{array}{l}209,268 \\
359\end{array}$ & 479 & $\begin{array}{l}\mathrm{MS}^{2}[479]: 317(100), 316(92.3), 179(16.0) \\
\mathrm{MS}^{3}[479 \rightarrow 317]: 287(30.6), 271(81.6), 193(40.6), 179(100) \\
\mathrm{MS}^{4}[479 \rightarrow 317 \rightarrow 179]: 169(100)\end{array}$ & myricetin-O-hexoside ${ }^{b}$ \\
\hline 23 & 7.7 & & 631 & $\begin{array}{l}\mathrm{MS}^{2}[631]: 479(39.7), 318(12.7), 317(100) \\
\mathrm{MS}^{3}[631 \rightarrow 317]: 179(100), 151(39.1) \\
\mathrm{MS}^{4}[631 \rightarrow 317 \rightarrow 179]: 169(28.6), 151(100)\end{array}$ & myricetin-O-(O-galloyl)hexoside \\
\hline 24 & 8.4 & & 597 & $\begin{array}{l}\mathrm{MS}^{2}[597]: 477(45.0), 459(15.3), 417(25.2), 388(17.3), 387(53.6) \\
\quad, 358(20.0), 357(100) \\
\mathrm{MS}^{3}[597 \rightarrow 357]: 300(15.3), 209(100), 123(12.1), 121(25.4) \\
\mathrm{MS}^{4}[597 \rightarrow 357 \rightarrow 209]: 191(70.5), 165(48.0), 147(100)\end{array}$ & glucaric acid derivative $^{b}$ \\
\hline 26 & 10.2 & $\begin{array}{l}207,265 \\
352\end{array}$ & 441 & $\begin{array}{l}\mathrm{MS}^{2}[441]: 289(100), 290(21.5), 169(17.5), 331(11.6) \\
\mathrm{MS}^{2}[441 \rightarrow 289]: 245(100), 179(28.3), 161(27.4), 135(17.6) \\
\quad 85(13.0) \\
\mathrm{MS}^{3}[441 \rightarrow 289 \rightarrow 245]: 203(100), 202(39.7), 204(65.1), 205(43.4) \\
\quad, 188(53.4)\end{array}$ & (epi) catechin-O-gallate ${ }^{b}$ \\
\hline 27 & 10.3 & 207,358 & 615 & $\begin{array}{l}\operatorname{MS}^{2}[615]: 302(14.7), 301(100), 313(16.6), 463 \\
\operatorname{MS}^{3}[615 \rightarrow 301]: 179(100), 193(15.5), 151(63.4) \\
\mathrm{MS}^{4}[615 \rightarrow 301 \rightarrow 179]: 257(11.5), 151(100), 169(64.3)\end{array}$ & galloylquercetin-O- hexoside ${ }^{b}$ \\
\hline 30 & 12.1 & $\begin{array}{c}208,267 \\
343\end{array}$ & 447 & $\begin{array}{l}\mathrm{MS}^{2}[447]: 285(91.8), 284(100), 255(22.0), 256(16.8) \\
\mathrm{MS}^{3}[447 \rightarrow 285]: 255(100), 227(42.3), 256(22.7), 257(17.4) \\
\quad 239(16.1), 223(103 .), 151(10.4) \\
\mathrm{MS}^{4}[447 \rightarrow 284 \rightarrow 255]: 255(16.2), 229(100), 227(25.7), 211(38.7) \\
\quad, 167(46.0)\end{array}$ & kaempferol-O-hexoside \\
\hline 33 & 13.4 & & 447 & $\begin{array}{l}\mathrm{MS}^{2}[447]: 301(100), 300(24.9), 302(14.8) \\
\mathrm{MS}^{3}[447 \rightarrow 301]: 273(22.8), 271(14.8), 255(10.7), 211(21.3) \\
\quad 179(76.6), 169(32.5), 151(100) \\
\mathrm{MS}^{4}[447 \rightarrow 301 \rightarrow 179]: 169(28.3), 151(100)\end{array}$ & quercetin-O-deoxyhexoside \\
\hline 35 & 15.3 & & 633 & $\begin{array}{l}\mathrm{MS}^{2}[633]: 488(16.5), 487(12.2), 470(18.7), 469(100), 347(50.7) \\
\mathrm{MS}^{3}[633 \rightarrow 469]: 347(76.0), 323(56.3), 303(20.4), 259(33.1) \\
\quad 235(15.7), 163(100), 145(50.9)\end{array}$ & $\begin{array}{l}\text { benzoyl-p-dicoumaryl- 2,7-anhydro-3- } \\
\text { deoxy-2-octulopyranosonic acid }{ }^{b}\end{array}$ \\
\hline
\end{tabular}


Table 2. continued

\begin{tabular}{|c|c|c|c|c|c|}
\hline no. & $t_{\mathrm{R}}(\min )$ & $\lambda_{\max }^{a}(\mathrm{~nm})$ & {$[\mathrm{M}-\mathrm{H}]^{-}(m / z)$} & HPLC-DAD-ESI $/ \mathrm{MS}^{n} m / z$ (\% base peak) & assigned identity \\
\hline 36 & 16.3 & $\begin{array}{l}211,267 \\
345\end{array}$ & 615 & $\begin{array}{l}\operatorname{MS}^{4}[633 \rightarrow 469 \rightarrow 163]: 119(100) \\
\operatorname{MS}^{2}[615]: 318(16.8), 317(100), 463(41.8) \\
\operatorname{MS}^{3}[615 \rightarrow 317]: 227(11.6), 193(16.2), 191(12.6), 180(12.4) \\
\quad 179(100), 151(33.3), 137(16.7)\end{array}$ & myricetin-O-(O-galloyl)deoxyhexoside \\
\hline 38 & 17.3 & $\begin{array}{c}211,267 \\
345\end{array}$ & 615 & $\begin{array}{l}\mathrm{MS}^{4}[615 \rightarrow 317 \rightarrow 179]: 151(100) \\
\operatorname{MS}^{2}[615]: 463(31.2) 318(13.2), 317(100) \\
\operatorname{MS}^{3}[615 \rightarrow 317]: 271(10.7), 255(10.9), 193(16.3), 192(15.5), \\
\quad 179(100), 151(60.7), 137(25.0)\end{array}$ & myricetin-O-(O-galloyl)deoxyhexoside \\
\hline 40 & 17.6 & & 431 & $\begin{array}{l}\mathrm{MS}^{4}[615 \rightarrow 317 \rightarrow 179]: 169(22.6), 151(100) \\
\mathrm{MS}^{2}[431]: 286(16.7), 285(100), 284(28.4), 255(10.5) \\
\mathrm{MS}^{3}[431 \rightarrow 285]: 257(61.7), 255(100), 239(32.5), 229(52.5), \\
\quad 197(30.7), 163(19.4)\end{array}$ & kaempferol-O-rhamnoside ${ }^{b}$ \\
\hline 46 & 26.6 & & 491 & $\begin{array}{l}\mathrm{MS}^{4}[431 \rightarrow 285 \rightarrow 255]: 229(46.7), 213(20.3), 189(13.3) \\
\quad 185(10.5), 151(100), 93(38.7) \\
\mathrm{MS}^{2}[491]: 371(12.9), 330(28.5), 330(20.3), 329(100), 314(10.7) \\
\mathrm{MS}^{3}[491 \rightarrow 329]: 315(53.0), 314(100) \\
\mathrm{MS}^{3}[491 \rightarrow 329 \rightarrow 314]: 300(40.3), 299(100)\end{array}$ & tricin-O-hexoside ${ }^{b}$ \\
\hline 49 & 29.1 & & 491 & $\begin{array}{l}\mathrm{MS}^{2}[491]: 473(20.8), 330(15.2), 329(100) \\
\mathrm{MS}^{3}[491 \rightarrow 329]: 314(100), 299(70.5), 271(52.1), 193(48.3), \\
\quad 181(62.0), 135(51.6)\end{array}$ & tricin-O-hexoside ${ }^{b}$ \\
\hline 50 & 29.4 & & 563 & $\begin{array}{l}\mathrm{MS}^{2}[563]: 356(11.0), 355(100) \\
\mathrm{MS}^{3}[563 \rightarrow 355]: 341(12.8), 340(100), 325(43.4) \\
\mathrm{MS}^{4}[563 \rightarrow 355 \rightarrow 340]: 326(15.2), 325(100), 296(79.8), 281(36.7) \\
\quad, 212(27.6)\end{array}$ & conidendrin-O-hexoside ${ }^{b}$ \\
\hline 52 & 30.4 & & 535 & $\begin{array}{l}\mathrm{MS}^{4}[563 \rightarrow 355 \rightarrow 325]: 296(100), 281(79.8), 212(27.6) \\
\mathrm{MS}^{2}[535]: 490(31.6), 489(100)\end{array}$ & $\begin{array}{l}\text { 5,7-dihydroxy-6,8-dimethoxyflavone-7- } \\
\text { O-glucuronide } b \text { (formate adduct) }\end{array}$ \\
\hline & & & & $\begin{array}{l}\mathrm{MS}^{3}[535 \rightarrow 489]: 327(46.0), 313(100), 298(55.5), 283(33.6) \\
\mathrm{MS}^{4}[535 \rightarrow 489 \rightarrow 313]: 298(100), 283(22.7), 269(32.4), 254(29.4)\end{array}$ & \\
\hline 54 & 31.9 & & 779 & $\operatorname{MS}^{2}[779]: 634(26.5), 633(100), 616(26.2), 615(74.3), 469(38.7)$ & $\begin{array}{l}\text { benzoyl-p-tricoumaryl- 2,7-anhydro-3- } \\
\text { deoxy-2-octulopyranosonic acid }\end{array}$ \\
\hline & & & & $\begin{array}{l}\mathrm{MS}^{3}[779 \rightarrow 633]: 488(18.7), 487 \text { (17.9), } 470 \text { (35.0), } 469 \text { (100), } \\
\quad 325(17.3), 265(11.1)\end{array}$ & \\
\hline & & & & $\mathrm{MS}^{4}[779 \rightarrow 633 \rightarrow 469]: 307(88.9), 163(100), 145(49.5)$ & \\
\hline 57 & 34.7 & & 327 & $\begin{array}{l}\mathrm{MS}^{2}[327]: 311(27.9), 294(13.1), 293(18.7), 229(100), 211(72.1) \\
\quad 183(14.5), 171(12.4) \\
\mathrm{MS}^{3}[327 \rightarrow 229]: 211(100), 209(32.3), 165(16.9), 127(32.2) \\
\quad 125(67.3)\end{array}$ & oxo-dihydroxy-octadecenoic acid ${ }^{b}$ \\
\hline
\end{tabular}

${ }^{a}$ Wavelengths not provided when the UV spectrum was not properly observed due to low intensity. ${ }^{b}$ Compound identified for the first time in Myrica genus.

other kaempferol conjugates have never been characterized in Myrica species.

Compound 27, with $[\mathrm{M}-\mathrm{H}]^{-}$at $m / z$ 615, was plausibly identified as galloylquercetin- $O$-hexoside after sequential loss of 152 and $162 \mathrm{Da}^{24}$ This compound has been previously characterized in tropical fruits, but not in Myrica.

Compound 51 exhibited $[\mathrm{M}-\mathrm{H}]^{-}$at $m / z 583$, and its $\mathrm{MS}^{2}$ fragmentation revealed kaempferol aglycone at $m / z 285$ (by loss of $298 \mathrm{Da}$ ). However, complete identification of $\mathbf{5 1}$ was not achieved, being characterized as a kaempferol derivative.

Compound 53 displayed $[\mathrm{M}-\mathrm{H}]^{-}$at $\mathrm{m} / z 677$ and $\mathrm{MS}^{2}$ base peak at $m / z 285$, due to the loss of $392 \mathrm{Da}$. Product ions at $\mathrm{m} / z 593$ and 575 corroborated kaempferol-O-rutinoside and dehydrated kaempferol-O-rutinoside, with $\mathbf{5 3}$ being identified as a kaempferol- $O$-rutinoside derivative.

Compound 43 exhibited a $[\mathrm{M}-\mathrm{H}]^{-}$ion at $m / z 565$ and a direct loss of $208 \mathrm{Da}(46+162 \mathrm{Da})$ that suggested a formate adduct plus hexoside moiety. Further fragmentation showed identical behavior as dichtomitin, an isoflavone, with product ions at $m / z 342\left[\mathrm{M}-\mathrm{H}-\mathrm{CH}_{3}\right]^{-}, 327\left[\mathrm{M}-\mathrm{H}-\mathrm{CH}_{3} \times 2\right]^{-}$, and $299\left[\mathrm{M}-\mathrm{H}-\mathrm{CH}_{3} \times 2-\mathrm{CO}\right]^{-25}$. Thus, 43 was plausibly classified as dichtomitin-O-hexoside, for the first time in Myrica.

Compounds 46 and 52 exhibited $[\mathrm{M}-\mathrm{H}]^{-}$ions at $\mathrm{m} / z 491$ and 535 and showed neutral losses of 162 and $208 \mathrm{Da}$, respectively. The sequential fragmentation allowed for the identification of two losses of $15 \mathrm{Da}$ each in both compounds, due to two methoxyl groups. Their fragmentation behaviors were consistent with those described before in herbs ${ }^{18}$ for tricin-O-hexoside (dihydroxy-dimethoxy- $O$-hexoside flavones) (46) and 5,7-dihydroxy-6,8-dimethoxy-7-O-glucuronide flavone (52) ${ }^{26}$ With longer retention times, compounds $47\left(t_{\mathrm{R}}=27.3\right.$ $\min )$ and $49\left(t_{\mathrm{R}}=29.1 \mathrm{~min}\right)$ exhibited similar fragmentation pattern as 46 , being also assigned as tricin-O-hexoside. On the basis of only $\mathrm{MS}^{n}$ data, the stereochemical structures of the sugar moieties could not be elucidated.

Compound 48 exhibited $[\mathrm{M}-\mathrm{H}]^{-}$ion at $\mathrm{m} / z 555$ and product ion at $\mathrm{m} / z 269$, due to loss of $286 \mathrm{Da}$. Further fragmentation produced characteristic ions of baicalein: at $\mathrm{m} / z$ $251\left[\mathrm{M}-\mathrm{H}_{2} \mathrm{O}-\mathrm{H}\right]^{-}, 241[\mathrm{M}-\mathrm{CO}-\mathrm{H}]^{-}$, and $223[\mathrm{M}-$ $\left.\mathrm{H}_{2} \mathrm{O}-\mathrm{CO}-\mathrm{H}\right]$, according to Han et al. ${ }^{27}$ Thus, 48 was 
<smiles>[R]c1ccc(-c2cc(=O)c3c(O)c([R])c(O)cc3o2)cc1[R]</smiles>

$\begin{array}{lll}\mathbf{R}_{\mathbf{1}} & \mathbf{R}_{\mathbf{2}} & \mathbf{R}_{3}\end{array}$

$\mathrm{H} \quad \mathrm{OH} \quad \mathrm{OH}$ Luteolin

$\mathrm{OH} \quad \mathrm{H} \quad \mathrm{H} \quad$ Baicalein<smiles>[R]c1cc(-c2oc3cc(O)cc(O)c3c(=O)c2O)cc([R])c1O</smiles>

\section{$\mathbf{R}_{1} \quad \mathbf{R}_{2}$ \\ $\mathrm{OH} \quad \mathrm{H}$ \\ $\mathrm{H} \quad \mathrm{H}$ \\ $\mathrm{OH} \quad \mathrm{OH}$ Myricetin}

Flavanols<smiles>Oc1cc(O)c2c(c1)OC(c1ccc(O)c(O)c1)C(O)C2</smiles>

(epi)catechin
Hydroxycinnamic acids $\quad \mathbf{R}$<smiles>[R]c1cc(/C=C/C(=O)O)ccc1O</smiles>

$\mathrm{OH} \quad$ Caffeic acid

$\mathrm{OCH}_{3} \quad$ Ferulic acid

$\mathrm{H} \quad p$-Coumaric acid
$\mathbf{R}$

H Cyanidin

$\mathrm{OH}$ Delphinidin<smiles>O=C(O)c1cc(O)c(O)c(O)c1</smiles>

Gallic acid

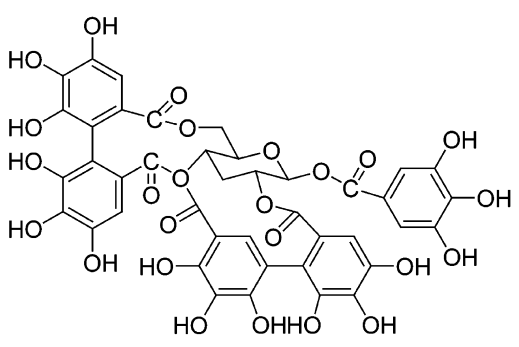

Galloyl-bis-HHDP-O-hexoside<smiles>COC(=O)C1=COC(OC2OC(CO)[C@H](O)C(O)[C@H]2O)[C@H](CC(=O)OCCc2ccc(O)c(O)c2)[C@H]1CO</smiles>

Oleuropein<smiles>COc1cc(-c2coc3cc4c(c(O)c3c2=O)OCO4)cc(O)c1O</smiles>

Dichotomitin

\section{Terpenoids}<smiles>COC1OC(OC(C)/C=C/[C@@]2(C)C(C)=CC(=O)CC2(C)OC)C(O)C(O)C1O</smiles>

Drovomifoliol- $O-B$-Dglucopyranoside

\section{Lignans}<smiles>COc1cc([C@H]2c3cc(O)c(OC)cc3C[C@@H]3C(=O)OC[C@@H]32)ccc1O</smiles>

Conidendrin<smiles>CC12CC3(COC(=O)c4ccccc4)C(=O)C3(C)CC1OC1O[C@H](CO)[C@@H](O)[C@H](O)[C@H]1O2</smiles>

Lactiflorin<smiles>COc1cc([C@@H]2OC[C@]3(C)[C@@H](c4ccc(OC)c(OC)c4)OC[C@]23C)ccc1O</smiles>

Phylligenin<smiles>O=C(O)C12OC(CO)C(O1)C(O)C(O)C2O</smiles>

2,7-anhydro-2-octulopyranosonic acid

Figure 3. Chemical structures of the main phytochemicals detected in the methanolic extracts from Myrica faya (leaves and berries).

identified as a baicalein derivative, detected for the first time in Myrica.
Compound 59 displayed $[\mathrm{M}-\mathrm{H}]^{-}$ion at $m / z 515$, and produced kaempferol aglycone at $m / z 284$ (by loss of $231 \mathrm{Da}$ ). 
In the absence of more specific data, $\mathbf{5 9}$ was characterized as a kaempferol derivative.

Lignans. Compounds $\mathbf{3 1}$ and $\mathbf{5 0}$ displayed $[\mathrm{M}-\mathrm{H}]^{-}$ions at $m / z 579$ and 563. In $\mathrm{MS}^{2}$ both compounds showed a loss of $208 \mathrm{Da}$ (possibly formic acid plus hexoside moieties). Further $\mathrm{MS}^{n}$ data were in accordance with those previously described in pomegranate for pylligenin and conidendrin. ${ }^{28}$ Thus, 31 and $\mathbf{5 0}$ were characterized as phylligenin- $O$-hexoside and conidendrin$O$-hexoside, respectively. To our best knowledge, we report here for the first time the presence of lignans in Myrica.

Other Compounds. Galloyl-bis-hexahydroxydiphenoyl(HHDP)-O-hexoside (compound 14) was plausibly identified in leaves according to previous findings. ${ }^{20}$ It showed $[\mathrm{M}-\mathrm{H}]^{-}$ ion at $\mathrm{m} / z 935$ and main fragment at $\mathrm{m} / z 633$ (HHDPgalloylhexoside) along with other product ions at $\mathrm{m} / z 615$ (dehydrated derivative), 481 (HHDP-hexoside), and 299 (ellagic acid). This finding marks the first report of an ellagitannin in Myrica species.

Additionally, some other nonphenolic compounds were detected in this analysis: organic acids, monoterpenes, phenylethanoids, and fatty acids.

Quinic acid showed $[\mathrm{M}-\mathrm{H}]^{-}$at $m / z 191$ (compound 5) and was plausibly characterized according to literature data. ${ }^{19}$ Quinic acid dimer (compound 7) with $[\mathrm{M}-\mathrm{H}]^{-}$at $\mathrm{m} / z 383$ showed a direct loss of $191 \mathrm{Da}$, with further fragmentation behavior identical to compound $\mathbf{5}$.

Compound 3, with $[\mathrm{M}-\mathrm{H}]^{-}$ion at $m / z 533$, exhibited a fragment ion at $m / z$ 191, which displayed the typical fragmentation pattern of quinic acid. Without further information, it was identified as a quinic acid derivative.

Benzyl alcohol hexose pentose (compound 15) displayed [ $\mathrm{M}$ $+\mathrm{HCOO}^{-}$ion at $\mathrm{m} / z$ 447, and sequential losses of 46 (formate) and 132 (pentose) Da were observed. This fragmentation pattern was similar to that previously described in Melicoccus bijugatus Jacq. fruits. ${ }^{29}$

Compound 16 exhibited $[\mathrm{M}+\mathrm{HCOO}]^{-}$ion at $\mathrm{m} / z 431$ and suffered the loss of $46 \mathrm{Da}$ (formate) to produce the ion at $\mathrm{m} / z$ 385 , which was identified as a roseoside (vomifoliolglucoside). It produced a fragment ion at $m / z 223$ by loss of a sugar moiety $(162 \mathrm{Da})$, and followed the exact behavior reported by Liet al. to what they called drovomifoliol-O-B-D-glucopyranoside (a terpenoid). ${ }^{30}$ Roseoside has been previously identified in Myrica's barks and leaves, but not in fruits. ${ }^{14}$

Another monoterpene was characterized (compound 45), with ions at $m / z 507\left[\mathrm{M}+\mathrm{HCOO}^{-}, 461[\mathrm{M}-\mathrm{H}]^{-}, 443[\mathrm{M}\right.$ $\left.-\mathrm{H}-\mathrm{H}_{2} \mathrm{O}\right]^{-}$, and $293\left[\mathrm{M}-\mathrm{H}-\mathrm{C}_{7} \mathrm{H}_{6} \mathrm{O}_{2}\right]^{-}$. It followed the exact same pattern described for lactiflorin, ${ }^{31}$ being documented here for the first time in this genus.

Compound 24 displayed an $[\mathrm{M}-\mathrm{H}]^{-}$ion at $m / z 597$, and its sequential fragmentation led to typical glucaric acid ions (at $m / z 209,191,147){ }^{32}$ Thus, with no other information available, $\mathbf{2 4}$ was identified as a glucaric acid derivative, reported here for the first time in Myrica.

The $\mathrm{MS}^{n}$ spectrum of compound 34 showed an $[\mathrm{M}-\mathrm{H}]^{-}$ ion at $\mathrm{m} / z 539$ which produced fragment ions at $\mathrm{m} / z 377$ [M $162-\mathrm{H}]^{-}$and $307\left[\mathrm{M}-162-\mathrm{C}_{4} \mathrm{H}_{6} \mathrm{O}-\mathrm{H}\right]^{-}$. Sequential fragmentation was consistent with that reported for oleuropein. ${ }^{33}$ This compound is one of the major phenolics (phenylethanoid) present in olive leaves and pulp and to our best knowledge have not been reported, so far, in Myrica.

Compound 35 exhibited $[\mathrm{M}-\mathrm{H}]^{-}$at $\mathrm{m} / z 633$ and during $\mathrm{MS}^{2}$ fragmentation lost $163 \mathrm{Da}$, which could be attributed to a coumaric acid. Further fragmentation gave product ions at $\mathrm{m} / z$
347 and 323, which corroborated the presence of benzoyl and coumaroyl groups (122 and $146 \mathrm{Da}$, respectively). This behavior was similar to that reported for benzoyl-p-coumaryl2,7-anhydro-3-deoxy-2-octulopyranosonic acid, ${ }^{34,35}$ with 35 being identified as this compound. With an extra neutral loss of $146 \mathrm{Da}$, compound 54 displayed $[\mathrm{M}-\mathrm{H}]^{-}$ion at $\mathrm{m} / z 779$ and was characterized as benzoyl-p-tricoumaryl-2,7-anhydro-3deoxy-2-octulopyranosonic acid. The presence of another coumaryl group instead of a rhamnose unit attached to the molecule was consistent with the longer retention time $\left(t_{\mathrm{R}}=\right.$ $31.9 \mathrm{~min}$ ). To our best knowledge, octulosonic acid derivatives were reported here, for the first time, in Myrica.

Compound 57 showed $[\mathrm{M}-\mathrm{H}]^{-}$ion at $\mathrm{m} / z$ 327. The neutral loss of $98 \mathrm{Da}$ in $\mathrm{MS}^{2}$ corresponded to the loss of an end-group $\mathrm{HO}-\mathrm{CH}=\mathrm{CH}\left(\mathrm{CH}_{2}\right)_{3} \mathrm{CH}_{3}$ from an oxylipin molecule. ${ }^{36}$ Compound $\mathbf{5 7}$ was thus identified as an oxodihydroxyoctadecenoic acid (oxo-DHODE). This compound, together with trihydroxyoctacedenoic acid (THODE), has been found by our group in the leaves of other species from Madeira endemic flora (unpublished results).

Other peaks (compounds 1, 41, 55, 56, and 58) were detected, but their UV and $\mathrm{MS}^{n}$ data did not provide any valuable information about their chemical nature. Thus, their structures could not be elucidated.

Positive Mode Ionization. Faya berries are red or dark in color, attributed mainly to anthocyanins, which are more easily characterized with electrospray ionization operating in the positive mode $\left(\mathrm{ESI}^{+}\right)$in combination to the characteristic UVDAD absorptions. ${ }^{20,21}$ The ESI $^{+}$analysis was only relevant for the berries extracts.

Compound 2 gave an $[\mathrm{M}+\mathrm{H}]^{+}$ion at $m / z 449$, and the main $\mathrm{MS}^{2}$ fragment ion was observed at $\mathrm{m} / z$ 287, corresponding to the neutral loss of $162 \mathrm{Da}$. Further fragmentation of the ion at $\mathrm{m} / z 287$ suggested that the aglycone was cyanidin based on literature data. ${ }^{13}$ Thus, 2 was characterized as cyanidin-3-glucoside, which has been reported as the dominant anthocyanin (95\% of total anthocyanins) present in Myrica rubra fruits.

Compound 9 exhibited $[\mathrm{M}-\mathrm{H}]^{-}$ion at $m / z 465$, forming a fragment ion at $m / z 303$ (by the loss of $162 \mathrm{Da}$ ). $\mathrm{MS}^{n}$ fragment ions at $\mathrm{m} / z 257$ and 229 were consistent with those reported for delphinidin. ${ }^{37}$ Therefore, 9 was characterized for the first time in Myrica as delphinidin-O-hexoside.

Quantification of Phenolic Compounds. In the present study, 21 polyphenols were quantified by HPLC-DAD using the corresponding standards for calibration for each group, and the obtained results are shown in Table 3 .

The phenolic composition of leaves and berries varied quantitatively. The results indicated that flavonols, flavanols, and phenolic acids were the most abundant compounds in the leaves. Myricetin-O-deoxyhexoside presented the highest concentration in leaves, which is in agreement with bibliographic data on $M$. rubra. ${ }^{11}$ Leaves were also rich in myricetin$\mathrm{O}$-(O-galloyl)deoxyhexoside, gallo(epi)catechin-O-gallate dimer, and galloyl-bis-HHDP-O-hexoside (casuarin). TIPC of the leaves was comparable to those reported previously in $M$. rubra (1133-2255 mg GAE/100 g of dried leaves).

For berries, anthocyanins, flavonols, and flavones represented the dominant class of polyphenols. C3G was the major compound, followed by myricetin- $O$-hexoside and luteolin- $O$ hexoside derivative. Previous work ${ }^{7,8,13}$ on juice and pomace from $M$. rubra also reported $\mathrm{C} 3 \mathrm{G}$ as one of the main polyphenols in berries. Flavonoids (in particular flavonols) 
Table 3. Contents of Total and Individual Phenolic Compounds (mg/100 $\mathrm{g} \mathrm{DW}$ ) in Leaves and Berries from Myrica faya $a^{a}$

\begin{tabular}{|c|c|c|}
\hline phenolic compounds & leaves & berries \\
\hline \multicolumn{3}{|l|}{ Phenolic Acids } \\
\hline caffeic acid- $O$-hexoside & & $45.73 \pm 0.97$ \\
\hline protocatechuic acid-O-pentoside & $7.53 \pm 0.9$ & \\
\hline total & $7.53 \pm 0.9$ & $45.73 \pm 0.97$ \\
\hline \multicolumn{3}{|l|}{ Flavanols } \\
\hline galloyl-di(epi)-gallocatechin & $43.42 \pm 0.98$ & \\
\hline (epi)catechin & $2.56 \pm 0.41$ & nd \\
\hline gallo(epi)catechin- $O$-gallate dimer & $132.06 \pm 8.27$ & \\
\hline (epi)catechin-O-gallate & & $37.80 \pm 1.22$ \\
\hline total & $178.04 \pm 4.33$ & $37.80 \pm 1.22$ \\
\hline \multicolumn{3}{|l|}{ Flavonols } \\
\hline myricetin- $O$-hexoside & $62.79 \pm 2.15$ & $80.98 \pm 2.65$ \\
\hline myricetin-O-(O-galloyl)hexoside & $53.59 \pm 1.59$ & nd \\
\hline myricetin-O-deoxyhexoside & $770.35 \pm 11.31$ & $44.20 \pm 1.23$ \\
\hline galloylquercetin-O-hexoside & $33.88 \pm 2.11$ & $41.73 \pm 2.06$ \\
\hline kaempferol-O-rutinoside & $13.25 \pm 0.85$ & - \\
\hline kaempferol-O-hexoside & $16.65 \pm 1.94$ & $8.44 \pm 0.46$ \\
\hline kaempferol-O-(O-galloyl)hexoside & $14.35 \pm 0.79$ & \\
\hline quercetin-O-deoxyhexoside & $16.86 \pm 0.86$ & $33.95 \pm 1.35$ \\
\hline $\begin{array}{l}\text { myricetin- } O \text { - }(O \text {-galloyl }) \\
\text { deoxyhexoside }\end{array}$ & $233.98 \pm 6.55$ & $59.51 \pm 2.64$ \\
\hline kaempferol-O-rhamnoside & $26.27 \pm 2.69$ & nd \\
\hline total & $1241.97 \pm 10.79$ & $268.81 \pm 9.43$ \\
\hline \multicolumn{3}{|l|}{ Flavones } \\
\hline luteolin- $O$-hexoside derivative & & $77.68 \pm 5.44$ \\
\hline tricin-O-hexoside & $26.38 \pm 1.48$ & $5.01 \pm 0.94$ \\
\hline total & $26.38 \pm 1.48$ & $82.69 \pm 4.79$ \\
\hline \multicolumn{3}{|l|}{ Ellagitannins } \\
\hline $\begin{array}{l}\text { galloyl-bis-HHDP-O-hexoside } \\
\text { (casuarin) }\end{array}$ & $86.46 \pm 6.75$ & \\
\hline total & $86.46 \pm 6.75$ & \\
\hline \multicolumn{3}{|l|}{ Anthocyanins } \\
\hline cyanidin-O-hexoside & & $368.57 \pm 5.42$ \\
\hline delphinidin-O-hexoside & & $16.52 \pm 0.36$ \\
\hline total & & $385.09 \pm 6.61$ \\
\hline TIPC & $1540.38 \pm 87.76$ & $820.11 \pm 46.71$ \\
\hline$a_{\text {nd }}=$ not detected. & & \\
\hline
\end{tabular}

were also present in higher amounts than phenolic acids. (epi)Catechin, myricetin-O-(O-galloyl)hexoside, and kaempferol-O-rhamnoside were not quantified in berries due to their low concentration.
The TIPC of leaves and berries was lower than those determined by the Folin-Ciocalteu method (Table 4). This difference is attributed to the fact that the Folin-Ciocalteu method tends to overestimate the contents of total phenolics, since it gives positive answer to other substances, and also because not all the identified compounds could be quantified.

Analysis of L-AA Content. The data regarding L-AA content, $\mathrm{pH}$, and ${ }^{\circ}$ Brix of faya berries are presented in Table 4 .

The amounts of L-AA present in $M$. faya berries had not been determined before and were within the range of those reported for bayberries (Table 4). No data were found about vitamin C content in M. esculenta. Apart from Myrica species, the L-AA contents obtained here were higher than others reported previously in other berry fruits like blackberry, blueberry, chokeberry, raspberry, and redcurrant, but lower than in blackcurrant and strawberry. ${ }^{17,38}$ The sugar content, evaluated through the ${ }^{\circ}$ Brix, was higher than in M. rubra and within the range reported for most commercial berries (usually between 10 and 18), and the acidity was low.

TPC, TFC, and Antioxidant Capacity Tests. The results obtained for total phenolic and flavonoid contents of Myrica faya leaves and berries are presented in Table 3. L-AA is a powerful antioxidant, and its presence in plant extracts produces inaccurate estimations of TPC values because L-AA reduces FCR. One approach to improve the TPC values is the calculation of a corrected TPC value based on the L-AA reducing activity present in the extract. ${ }^{39,40}$ The L-AA standard was tested for TPC using the same procedure previously described and it was found to present reducing activity of 0.683 $\mathrm{mg}$ GAE/g L-AA. So, for each sample, L-AA contribution was calculated by multiplying the L-AA content by 0.683 . The corrected TPC of the samples, calculated by subtracting L-AA contribution, is also shown in Table 4.

Our tests revealed that leaves had a much higher content of total phenolics than berries, which is in agreement with previous literature reports for other subspecies of Myrica. TPC values of Myrica faya (leaves and berries) are comparable to those reported for other Myrica species (with faya showing a slightly higher content). Compared with other commonly consumed berries, faya presented higher TPC values than blackberry, blueberry, raspberry, and strawberry. ${ }^{17,38}$ For TFC assay the same pattern was observed; however, no data regarding flavonoid content of leaves was found in the literature for comparison. Rawat et al. reported TFC values of $M$. esculenta (131-238 mg quercetin equivalent/100 $\mathrm{g}$ of dried weight), but those results were expressed in different units, which makes a comparison difficult. ${ }^{15}$

Table 4. Overview of L-AA, pH, TSS, TPC, TFC, and Antioxidant Capacity Assays (ABTS, DPPH) Determinations in Myrica faya (Leaves and Berries) ${ }^{a}$

\begin{tabular}{|c|c|c|c|c|c|c|}
\hline \multirow[b]{2}{*}{ parameters } & \multicolumn{2}{|c|}{ faya (Myrica faya) } & \multicolumn{2}{|c|}{ bayberry (Myrica rubra) } & \multicolumn{2}{|c|}{ box myrtle (Myrica esculenta) } \\
\hline & leaves & berries & leaves & berries & leaves & berries \\
\hline $\mathrm{pH}$ & & 4.02 & & & & \\
\hline TSS $\left({ }^{\circ}\right.$ Brix $)$ & & 14.87 & & $11.6-13.4^{41}$ & & \\
\hline L-AA content (mg/100 g FW) & & $48.42 \pm 1.93$ & & $11-114^{41}$ & 4.16 & \\
\hline TPC (g GAE/100 g DE) & $24.80 \pm 0.28$ & $5.26 \pm 0.13$ & $8.14-19.63^{11}$ & $0.07-4.74^{6-8,12,13}$ & $15^{15}$ & $0.18-2.86^{15,16}$ \\
\hline $\mathrm{TPC}(\mathrm{g} \mathrm{GAE} / 100 \mathrm{~g} \mathrm{DE})^{b}$ & & $5.23 \pm 0.13$ & & & & \\
\hline TFC (g RUE/100 g DE) & $12.72 \pm 0.16$ & $4.21 \pm 0.12$ & & $0.01-0.12^{6,12}$ & & \\
\hline ABTS (g TE/100 g DE) & $24.10 \pm 0.13$ & $12.51 \pm 0.15$ & & $0.33-10.06^{6,8,12}$ & $38.45^{15}$ & \\
\hline DPPH (g TE/100 g DE) & $20.36 \pm 0.12$ & $9.24 \pm 0.11$ & & $0.74-9.10^{12,13}$ & & \\
\hline
\end{tabular}

${ }^{a}$ All measurements were performed in triplicate (mean $\left.\pm \mathrm{SD}\right) .{ }^{b}$ corrected TPC value (subtracted L-AA contribution). 
In this study, both ABTS and DPPH were used to evaluate the antioxidant capacity of Myrica faya, and the results are shown in Table 4. Myrica faya presented a considerable freeradical scavenging capacity, with leaves showing a stronger reducing power than fruits, which corroborated the measured phenolic and flavonoid contents.

The values obtained for $M$. faya in the ABTS assay were slightly higher than the range of values reported for M. rubra, but lower than those from M. esculenta. Faya berries were much more active than, for instance, strawberries $(1455.50 \mu \mathrm{mol} \mathrm{TE})$ evaluated in the same experimental conditions (data to be published elsewhere). According to Sun et al., ${ }^{9}$ many structure-activity relationship studies have confirmed that the strong antioxidant capacities of Myrica species are attributed to the high content of galloyl esters that enhance such properties and confer high radical scavenging activities. ${ }^{9}$

In conclusion, over 50 compounds were characterized, for the first time, in different morphological parts of Myrica faya by means of an HPLC-DAD-ESI/MS ${ }^{n}$ method. M. faya shared some characteristics in phenolic profile with other Myrica species. Nevertheless, we reported for the first time the presence of some flavonoids, ellagitannins, lignans, phenylethanoids, and other organic compounds in this genus. The levels of L-AA and C3G observed in the berries were high, so they can constitute a good source of these nutrients when compared to other fruits. This study provides scientific evidence that $M$. faya is a rich source of bioactive compounds with great potential as natural antioxidants. Faya berries are underutilized, mainly due to the lack of scientific studies about their potential health benefits, and consumption and marketing deserve promotion, representing an opportunity for growers and collectors to reach niche markets to increase their revenues.

\section{AUTHOR INFORMATION}

\section{Corresponding Author}

*Phone: +351 291705 102. E-mail: castilho@uma.pt.

\section{Funding}

V.S. is grateful to Fundação para a Ciência e Tecnologia (FCT, Portugal) for a Ph.D. grant SFRH/BD/84672/2012. This research was supported by FCT with funds from the Portuguese Government (Project PEst-OE/QUI/UI0674/ 2011). The mass spectrometer used in this work is part of the Portuguese National Mass Spectrometry Network (Contract RNEMREDE/1508/REM/2005) and was purchased in the framework of the National Programme for Scientific Reequipment, with funds from POCI 2010 (FEDER) and FCT. E.J.L.-M. also acknowledges the financial support from Campus de Excelencia Internacional Agroalimentario (ceiA3) and University of Jaén, from Spain.

\section{Notes}

The authors declare no competing financial interest.

\section{ABBREVIATIONS USED}

FCR, Folin-Ciocalteu's phenol reagent; trolox, 6-hydroxy2,5,7,8-tetramethylchroman-2-carboxylic acid; ABTS, 2,2'azinobis(3-ethylbenzthiazoline-6-sulfonic acid); DPPH, 2,2diphenyl-1-picrylhydrazyl; MPA, metaphosphoric acid; EDTA, ethylenediaminetetraacetic acid disodium salt; $\mathrm{DAD}$, diodearray detector; $\mathrm{AR}$, analytical reagent; L-AA, L-ascorbic acid; $\mathrm{CH}_{3} \mathrm{CN}$, acetonitrile; TSS, total soluble solids; $\mathrm{Na}_{2} \mathrm{CO}_{3}$, sodium carbonate; $\mathrm{CH}_{3} \mathrm{COOK}$, sodium acetate; $\mathrm{AlCl}_{3} \cdot 6 \mathrm{H}_{2} \mathrm{O}$, aluminum chloride hexahydrated; PBS, phosphate buffered saline; DE, dried extract; ANOVA, analysis of variance; TE, trolox equivalent; RUE, rutin equivalent; GA, gallic acid equivalent; HPLC-DAD-ESI/MS ${ }^{n}$, high performance liquid chromatography with online UV and electrospray ionization mass spectrometric detection; SD, standard deviation

\section{REFERENCES}

(1) Amira, A. M. A. Oxidative stress and disease: An updated review. Res. J. Immunol. 2011, 3, 129-145.

(2) Gouveia, S.; Spínola, V.; Castilho, P. C., Phenolic compounds and antioxidant capacity of medicinal plants: A review. In Medicinal Plants: Antioxidant Properties, Traditional Uses and Conservation Strategies; Nova Science Publishers, Inc.: Hauppauge, NY, 2013; pp 1-40.

(3) Shan, B.; Cai, Y.-Z.; Brooks, J. D.; Corke, H. The in vitro antibacterial activity of dietary spice and medicinal herb extracts. Int. J. Food Microbiol. 2007, 117, 112-119.

(4) GISD (Global Invasive Species Database): Morella faya (tree, shrub). http://www.issg.org/database/species/ecology.asp?si=100 (accessed Sept 20 2013).

(5) Pérez, M. Á. C. Native Flora of the Canary Islands. Editorial Everest: Leon, Spain, 2000.

(6) Bao, J.; Cai, Y.; Sun, M.; Wang, G.; Corke, H. Anthocyanins, flavonols, and free radical scavenging activity of Chinese bayberry (Myrica rubra) extracts and their color properties and stability. J. Agric. Food Chem. 2005, 53, 2327-2332.

(7) Fang, Z.; Zhang, M.; Wang, L. HPLC-DAD-ESIMS analysis of phenolic compounds in bayberries (Myrica rubra Sieb. et Zucc.). Food Chem. 2007, 100, 845-852.

(8) Fang, Z.; Zhang, Y.; Lüa, Y.; Ma, G.; Chen, J.; Liu, D.; Ye, X. Phenolic compounds and antioxidant capacities of bayberry juices. Food Chem. 2009, 113, 884-888.

(9) Sun, C.; Huang, H.; Xu, C.; Li, X.; Chen, K. Biological activities of extracts from Chinese bayberry (Myrica rubra Sieb. et Zucc.): A review. Plant Foods Hum. Nutr. 2013, 68, 97-106.

(10) Wang, C.; Zhao, J.; Chen, F.; Cheng, Y.; Guo, A. Separation, identification, and quantitation of phenolic acids in Chinese waxberry (Myrica rubra) Juice by HPLC-PDA-ESI-MS. J. Food Sci. 2012, 77, C272-C277.

(11) Yang, H.; Ge, Y.; Sun, Y.; Liu, D.; Ye, X.; Wu, D. Identification and characterisation of low-molecular-weight phenolic compounds in bayberry (Myrica rubra Sieb. et Zucc.) leaves by HPLC-DAD and HPLC-UV-ESIMS. Food Chem. 2011, 128, 1128-1135.

(12) Zhang, W.; Li, X.; Zheng, J.; Wang, G.; Sun, C.; Ferguson, I.; Chen, K. Bioactive components and antioxidant capacity of Chinese bayberry (Myrica rubra Sieb. and Zucc.) fruit in relation to fruit maturity and postharvest storage. Eur. Food Res. Technol. 2008, 227, 1091-1097.

(13) Zhou, S. H.; Fang, Z. X.; Lü, Y.; Chen, J. C.; Liu, D. H.; Ye, X. Q. Phenolics and antioxidant properties of bayberry (Myrica rubra Sieb. et Zucc.) pomace. Food Chem. 2009, 112, 394-399.

(14) Panthari, P.; Kharkwal, H.; Joshi, D. D. Myrica nagi: A review on active constituents, biological and therapeutic effects. Int. J. Pharm. Pharm. Sci. 2012, 4, 38-42.

(15) Rawat, S.; Jugran, A.; Giri, L.; Bhatt, I. D.; Rawal, R. S. Assessment of antioxidant properties in fruits of Myrica esculenta: A popular wild edible species in Indian Himalayan region. J. EvidenceBased Complementary Altern. Med. 2011, 2011.

(16) Saini, R.; Garg, V.; Dangwal, K. Effect of extraction solvents on polyphenolic composition and antioxidant, antiproliferative activities of Himalyan bayberry (Myrica esculenta). Food Sci. Biotechnol. 2013, 22, 887-894.

(17) Spínola, V.; Mendes, B.; Câmara, J. S.; Castilho, P. C. Effect of time and temperature on vitamin $\mathrm{C}$ stability in horticultural extracts. UHPLC-PDA vs iodometric titration as analytical methods. LWTFood Sci. Technol. 2013, 50, 489-495.

(18) Gouveia, S.; Castilho, P. C. Antioxidant potential of Artemisia argentea L'Hér alcoholic extract and its relation with the phenolic composition. Food Res. Int. 2011, 44, 1620-1631. 
(19) Gouveia, S.; Castilho, P. C. Characterisation of phenolic acid derivatives and flavonoids from different morphological parts of Helichrysum obconicum by a RP-HPLC-DAD-(-)-ESI-MS ${ }^{\mathrm{n}}$ method. Food Chem. 2011, 129, 333-344.

(20) Fischer, U. A.; Carle, R.; Kammerer, D. R. Identification and quantification of phenolic compounds from pomegranate (Punica granatum L.) peel, mesocarp, aril and differently produced juices by HPLC-DAD-ESI/MS . Food Chem. 2011, 127, 807-821.

(21) Mena, P.; Calani, L.; Dall'Asta, C.; Galaverna, G.; GarcíaViguera, C.; Bruni, R.; Crozier, A.; Del Rio, D. Rapid and comprehensive evaluation of (poly)phenolic compounds in pomegranate (Punica granatum L.) juice by UHPLC-MSn. Molecules 2012, 17, 14821-14840.

(22) Calani, L.; Beghè, D.; Mena, P.; Del Rio, D.; Bruni, R.; Fabbri, A.; Dall'Asta, C.; Galaverna, G. Ultra-HPLC-MSn (poly)phenolic profiling and chemometric analysis of juices from ancient Punica granatum L. Cultivars: A nontargeted approach. J. Agric. Food Chem. 2013, 61, 5600-5609.

(23) Rockenbach, I. I.; Jungfer, E.; Ritter, C.; Santiago-Schübel, B.; Thiele, B.; Fett, R.; Galensa, R. Characterization of flavan-3-ols in seeds of grape pomace by CE, HPLC-DAD-MS ${ }^{\mathrm{n}}$ and LC-ESI-FTICRMS. Food Res. Int. 2012, 48, 848-855.

(24) Gordon, A.; Jungfer, E.; da Silva, B. A.; Maia, J. G. S.; Marx, F. Phenolic constituents and antioxidant capacity of four underutilized fruits from the Amazon region. J. Agric. Food Chem. 2011, 59, 76887699.

(25) Zhang, Y.-Y.; Wang, Q.; Qi, L.-W.; Qin, X.-Y.; Qin, M.-J. Characterization and determination of the major constituents in Belamcandae Rhizoma by HPLC-DAD-ESI-MS ${ }^{\mathrm{n}}$. J. Pharm. Biomed. Anal. 2011, 56, 304-314.

(26) Li, J.; Wang, Y.-H.; Smillie, T. J.; Khan, I. A. Identification of phenolic compounds from Scutellaria lateriflora by liquid chromatography with ultraviolet photodiode array and electrospray ionization tandem mass spectrometry. J. Pharm. Biomed. Anal. 2012, 63, 120127.

(27) Han, J.; Ye, M.; Xu, M.; Sun, J.; Wang, B.; Guo, D. Characterization of flavonoids in the traditional Chinese herbal medicine-Huangqin by liquid chromatography coupled with electrospray ionization mass spectrometry. J. Chromatogr. B: Anal. Technol. Biomed. Life Sci. 2007, 848, 355-362.

(28) Fischer, U. A.; Jaksch, A. V.; Carle, R; Kammerer, D. R. Determination of lignans in edible and nonedible parts of pomegranate (Punica granatum L.) and products derived therefrom, particularly focusing on the quantitation of isolariciresinol Using HPLC-DADESI/MS . J. Agric. Food Chem. 2012, 60, 283-292.

(29) Bystrom, L. M.; Lewis, B. A.; Brown, D. L.; Rodriguez, E.; Obendorf, R. L. Characterisation of phenolics by LC-UV/Vis, LCMS/MS and sugars by GC in Melicoccus bijugatus Jacq. 'Montgomery' fruits. Food Chem. 2008, 111, 1017-1024.

(30) Li, X.; Zhang, Y.; Zeng, X.; Yang, L.; Deng, Y. Chemical profiling of bioactive constituents in Sarcandra glabra and its preparations using ultra-high-pressure liquid chromatography coupled with LTQ Orbitrap mass spectrometry. Rapid Commun. Mass Spectrom. 2011, 25, 2439-2447.

(31) Xu, S.-j.; Yang, L.; Zeng, X.; Zhang, M.; Wang, Z.-t. Characterization of compounds in the Chinese herbal drug $M u-D a n-$ $P i$ by liquid chromatography coupled to electrospray ionization mass spectrometry. Rapid Commun. Mass Spectrom. 2006, 20, 3275-3288.

(32) Simirgiotis, M. J.; Caligari, P. D. S.; Schmeda-Hirschmann, G. Identification of phenolic compounds from the fruits of the mountain papaya Vasconcellea pubescens A. DC. grown in Chile by liquid chromatography-UV detection-mass spectrometry. Food Chem. 2009, 115, 775-784.

(33) Savarese, M.; De Marco, E.; Sacchi, R. Characterization of phenolic extracts from olives (Olea europaea cv. Pisciottana) by electrospray ionization mass spectrometry. Food Chem. 2007, 105, 761-770.

(34) Wang, Y.-F.; Hu, L.-M.; Liu, Y.-N.; Pan, X.-P.; Pan, G.-X.; Chang, Y.-X.; Gao, X.-M. A rapid method for qualitative and quantitative analysis of major constituents in Dengzhanxixin injection by LC-DAD-ESI-MS ${ }^{\mathrm{n}}$. Chromatographia 2010, 71, 845-853.

(35) Zhang, Y.; Shi, P.; Qu, H.; Cheng, Y. Characterization of phenolic compounds in Erigeron breviscapus by liquid chromatography coupled to electrospray ionization mass spectrometry. Rapid Commun. Mass Spectrom. 2007, 21, 2971-2984.

(36) Van Hoyweghen, L.; De Bosscher, K.; Haegeman, G.; Deforce, D.; Heyerick, A. In vitro inhibition of the transcription factor NF- $\kappa$ B and cyclooxygenase by bamboo extracts. Phytother. Res. 2014, 28, 224-230.

(37) Lätti, A. K.; Riihinen, K. R.; Jaakola, L. Phenolic compounds in berries and flowers of a natural hybrid between bilberry and lingonberry (Vaccinium $\times$ intermedium Ruthe). Phytochemistry 2011, 72, 810-815.

(38) Szajdek, A.; Borowska, E. J. Bioactive compounds and healthpromoting properties of berry fruits: A review. Plant Foods Hum. Nutr. 2008, 63, 147-156.

(39) Isabelle, M.; Lee, B. L.; Lim, M. T.; Koh, W.-P.; Huang, D.; Ong, C. N. Antioxidant activity and profiles of common vegetables in Singapore. Food Chem. 2010, 120, 993-1003.

(40) Sanchez-Rangel, J. C.; Benavides, J.; Heredia, J. B.; CisnerosZevallos, L.; Jacobo-Velazquez, D. A. The Folin-Ciocalteu assay revisited: improvement of its specificity for total phenolic content determination. Anal. Methods 2013, 5, 5990-5999.

(41) Chen, K.; Xu, C.; Zhang, B.; Ferguson, I. B. Red bayberry: Botany and horticulture. In Horitcultural Reviews; Janick, J., Ed.; John Wiley \& Sons: New York, 2004. 